\title{
RESOLUCIÓN DE ALGUNOS PROBLEMAS ESTOCÁSTICOS MEDIANTE DISCRETIZACIÓN
}

\section{(THE SOLUTION OF SOME STOCHASTIC PROBLEMS BY DISCRETIZATION)}

José L. Romero Martín, Dr. Ingeniero de Caminos, Profesor Fac. Informática. Emilio de la Rosa Oliver, Dr. Ingeniero de Caminos, Catedrático E.T.S.I.C.C. y P.

Universidad Politécnica de Madrid - España

$406-4$

\section{RESUMEN}

El propósito del presente trabajo es exponer una nueva técnica de resolución aproximada de problemas estocásticos $y$, dentro de ellos, los relacionados especialmente con las ecuaciones diferenciales estocásticas.

La aplicación del método propuesto a varios casos ha permitido destacar las posibilidades del mismo en relación con métodos clásicos tales como los de Taylor y Monte Carlo.
SUMMARY

The aim of this work, is to show a new aproximate resolution technique for stochastic problems and among them, those related to stochastic differential equations.

The application of the proposed method to several cases has allowed to emphasize its possibilities in relation to other classical methods such as Taylor and Monte Carlo methods.

\section{Introducción}

La formulación estocástica de muchos modelos físicos supone en general un gran avance respecto a los clásicos esquemas deterministas. Sin embargo la contrapartida de estos modelos es la de mayor complejidad en la resolución de los problemas a que dan lugar, de ahi que tenga gran interés desarrollar métodos aproximados para la resolución de los mismos.

Supongamos que $Y$ es la incógnita del problema y $X_{1}$, $X_{2}, \ldots, X_{q}$ los datos (coeficientes, parámetros, etc.) del mismo. La relación entre dichas variables puede expresarse asi:

$$
Y=g\left(X_{1}, X_{2}, \ldots, X_{q}\right)
$$

Si las $X_{i}, i=1, \ldots, q$ son variables aleatorias $X_{i}(\omega)$ y g una función medible de las mismas, entonces $Y$ es una variable aleatoria.
Una situación de especial interés es aquella en la que g no está dada explícitamente, como ocurre por ejemplo en el siguiente problema de contorno:

$$
\left\{\begin{array}{l}
y^{\prime \prime}+x_{1}(\omega) q(x) y=x_{2}(\omega) r(x) \\
y(0)=0 \\
y(1)=0
\end{array}\right.
$$

en el cual podemos considerar que la variable aleatoria $Y$ corresponde al valor que toma el proceso solución $\mathrm{y}(\mathrm{x}, \omega)$ en un punto particular $\mathrm{x}_{0} \epsilon[0,1]$.

El propósito en este tipo de estudios es obtener tanta información como sea posible de la variable $Y$ sin necesidad de conocer la expresión exacta de la dependencia g, la cual independientemente es imposible obtener en la mayoría de los casos. 
Por otra parte el estudio es más complicado cuando los problemas contienen no sólo variables aleatorias, sino procesos estocásticos entre sus parámetros; en estos casos el objetivo suele limitarse a la obtención aproximada de los primeros momentos de $\mathrm{Y}$, especialmente media y varianza siendo esto suficiente para la mayoria de las aplicaciones técnicas.

Asimismo puede indicarse que se han desarrollado muy diversos métodos, en cuanto a técnicas empleadas se refiere, debido por otro lado a la gran variedad de tipos de ecuaciones que suelen plantearse en dichos modelos estocásticos. En relación con esto, pueden destacarse las publicaciones: Bharucha-Reid [4], [5], [6] Boyce [7] y Sheidt y Purkert [15].

Por nuestra parte en el presente trabajo, y con una linea diferente a las de las publicaciones anteriormente citadas, aportamos un método original basado en la discretización de las variables aleatorias, datos que intervienen en el problema. Esta idea de discretización permite tratar desde el punto de vista numérico y de manera sistemática un conjunto muy amplio de problemas, entre los cuales se encuentran los de contorno y los de autovalores estocásticos.

En concreto el procedimiento consiste en asociar a cada una de las variables aleatorias datos, una sucesión de variables aleatorias discretas y finitas cuyos momentos convergen a los de las primeras. Para ello se elige un tipo especial de discretización, que ofrece la posibilidad de aproximar además en condiciones bastante generales los momentos de variables aleatorias que son función de las variables aleatorias datos, e incluso aproximar sus funciones de distribución exactas mediante otras construidas a partir de las funciones de distribución de las variables discretizadas.

Los fundamentos teóricos del método que proponemos podemos enmarcarlos dentro de la teoría de polinomios ortogonales y particularmente en la cuadratura de Gauss.

El método, por otra parte, guarda una cierta analogía con el de Monte Carlo al basar la resolución del problema estocástico en la resolución de una familia de problemas deterministas los cuales resultan, en este caso, de considerar las distintas combinaciones de valores de las variables discretizadas.

Es interesante indicar también, que para la aplicación del método no es necesario conocer la distribución exacta de las variables datos sino simplemente los primeros momentos, los cuales pueden ser estimados a partir de datos obtenidos experimentalmente (histogramas).
Por otro lado y en cuanto a la técnica de discretización se refiere, podemos señalar que la conexión de nuestro planteamiento con la teoría de polinomios ortogonales nos ha permitido generalizar los resultados expuestos por Rosenblueth en [14].

Finalmente destacar que en este trabajo no se pretende exponer con todo detalle los desarrollos teóricos necesarios, sino que el objetivo es más bien dar una visión global del método indicando, con varios ejemplos, las posibilidades que ofrece y el alcance del mismo.

\section{Generación de distribuciones discretas de variables aleatorias basada en la teoría de la cuadratura de Gauss.}

Los métodos de Taylor y de Monte Carlo (v. Benjamín y Cornell [3] y Ang y Tang [1], [2]) son los que habitualmente se emplean en la resolución aproximada de problemas estocásticos a pesar de que tienen ciertas limitaciones: En primer lugar el método de Taylor exige para su aplicación la existencia y continuidad de cierto número de derivadas de la función g para poder aproximar los momentos de la variable aleatoria

$$
Y=g\left(X_{1}, X_{2}, \ldots X_{q}\right)
$$

en función de los momentos de las variables $X_{1}$, $X_{2}, \ldots, X_{q}$. En segundo lugar la aplicación de este método a problemas estocásticos para los que g no se conoce ni explícita ni implícitamente (problemas de contorno, autovalores, etc.) exige de recursos adicionales como las técnicas de perturbación (v. Boyce [7] y Haines [10]), dando lugar en estos casos a una aplicación muy laboriosa del método.

Por otro lado, la aplicación del método de Monte Carlo evita algunos de los inconvenientes del método de Taylor (cálculo de derivadas, técnica de perturbación, etc.), pero la limitación que tiene este método, respecto de sus conocidas ventajas, es necesitar el empleo de muestras de gran tamaño para que sus resultados puedan considerarse aceptables.

Frente a los inconvenientes de los métodos anteriores destacamos el método de discretización, el cual permite dar un tratamiento computacional sencillo a estos problemas, al basarse en la discretización de las variables aleatorias que intervienen como datos o parámetros. Esta idea de discretización se expone seguidamente aplicada a un problema particular de pandeo estocástico.

Sea una pieza recta de longitud $L$ y rigidez variable $E l(x)$ empotrada rígidamente en $\mathrm{x}=0$ y elásticamente en $x=L$, siendo la constante de empotramiento elástico la variable aleatoria $\mathrm{k}(\omega)$. (Fig. 2.1). 


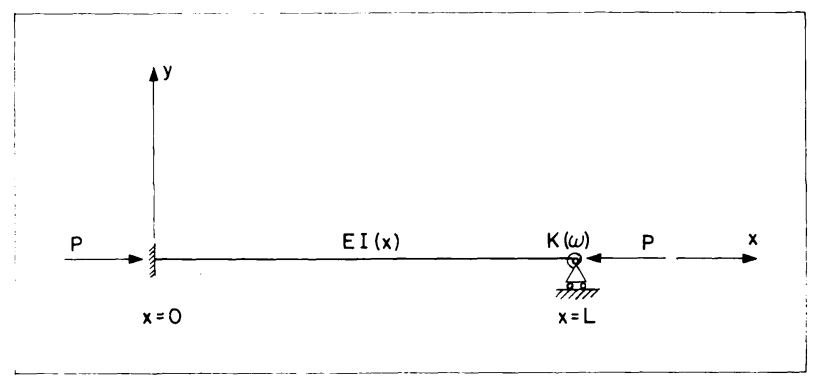

Fig. 2.1

Obsérvese que la función g, que relaciona la variable aleatoria $P_{c r}(\omega)$ (carga de pandeo) con la variable aleatoria $K(\omega)$, es decir $P_{c r}=g(K)$, está definida mediante el problema de autovalores estocásticos:

$$
\left[P_{K}(\omega)\right]\left\{\begin{array}{l}
\left(E I(x) y^{\prime}\right) "+P y^{\prime \prime}=0 \\
y(0)=0 \\
y^{\prime}(0)=0 \\
y(L)=0 \\
E I(L) y^{\prime \prime}(L)+K(\omega) y^{\prime}(L)=0
\end{array}\right.
$$

no siendo posible una expresión explícita de la misma ni aún en el caso más simple en que $\mathrm{El}(\mathrm{x})=$ cte.

El procedimiento que proponemos para aproximar las características estocásticas de $\mathrm{P}_{\mathrm{cr}}$ consiste en asociar a $\mathrm{K}$ la variable aleatoria discreta finita $K_{n}$ :

$$
K_{n}=\left(\begin{array}{cccc}
P_{n 1} & P_{n 2} & \cdots & P_{n n} \\
t_{n 1} & t_{n 2} & \cdots & t_{n n}
\end{array}\right)
$$

donde,

$$
\text { Prob }\left\{k_{n}=t_{n i}\right\}=p_{n i}>0 ; i=1, \ldots, n, \quad \sum_{i=1}^{n} p_{n i}=1,
$$

obteniéndose los puntos de discretización tni y las probabilidades respectivas $p_{n i}$ a partir de la distribución de la variable aleatoria $\mathrm{K}$.

De este modo al problema estocástico $\left[\mathrm{P}_{\mathrm{K}(\omega)}\right]$ podemos asociarle la familia de problemas deterministas $\left[\mathrm{P}_{\mathrm{t} \mathrm{ni}}\right]$ que resultan al sustituir en (2.1) la variable $\mathrm{K}(\omega)$ por cada una de las constantes $t_{n i}$. La resolución numérica de cada uno de estos problemas permitirá obtener los valores

$$
P_{a_{n i}}=g\left(t_{n i}\right)
$$

asociados cada uno de ellos a las probabilidades $p_{n i}$. Todo lo anterior da lugar a que a la variable aleatoria $\mathrm{P}_{\mathrm{cr}}(\omega)$ se le pueda asociar la variable aleatoria discreta $P_{C r_{n}}=g\left(K_{n}\right)$, donde:

$$
P_{c r_{n}}=\left(\begin{array}{llll}
P_{n 1} & P_{n 2} & \cdots & P_{n n} \\
P_{c r_{n 1}} & P_{c_{n n}} & \cdots & P_{c_{n n}}
\end{array}\right)
$$

Este proceso puede esquematizarse (Fig. 2.2) para el caso general de dos variables aleatorias $X$ e $Y$ donde $Y=g(X)$ siendo $g$ una función medible de $X$. En dicho proceso el paso (0) es sustituido por (1), (2), y (3).

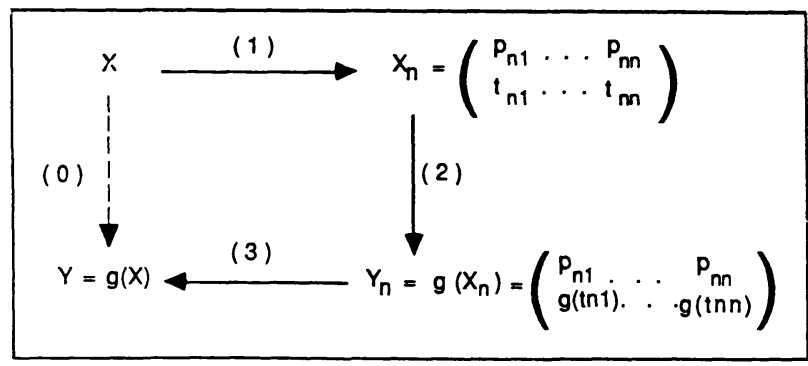

Fig. 2.2.

Obsérvese que el esquema es análogo al de Monte Carlo, considerando que la realización de una muestra de tamaño $n$ para la variable aleatoria $X$ podría interpretarse como el conjunto de valores que toma la variable discreta $X_{n}$ donde cada valor lleva asociada la probabilidad $1 / n$.

Sin embargo, nuestro propósito es elegir una sucesión de variables aleatorias discretas $\left\{X_{n}\right\}_{n \in I N}$ de modo que para cada $n$ fijo las variables $X$ y $X_{n}$ tengan en común el mayor número posible de momentos, con objeto de que $X_{n}$ "almacene" la mayor cantidad de información estadística posible (en el sentido de los momentos) de la variable $X$.

Esta condición de igualdad de momentos hasta el de mayor orden posible origina, en cierto modo, un tipo "óptimo" de discretización que asegura además la convergencia en distribución de la sucesión de variables aleatorias $\left\{Y_{n}\right\}_{n \in I N}$ a la variable $Y$, fundamentalmente en los casos en que $Y$ sea acotada, situación por otra parte muy frecuente en las aplicaciones.

El estudio de la igualdad de momentos entre $X$ y $X_{n}$ y el análisis de la convergencia en distribución de la sucesión $\left\{Y_{n}\right\} n_{n} \in \mathbb{N}$ a Y, y en particular de la convergencia de momentos, es decir:

$$
\lim _{n \rightarrow \infty} E\left[Y_{n}^{k}\right]=E\left[Y^{k}\right]
$$

para cada $k$ (fijo) $\epsilon$ IN permite conectar todo este planteamiento con algunos aspectos de la teoría de integración numérica, particularmente con la teoría de polinomios ortogonales y la cuadratura de Gauss.

A continuación se exponen esquemáticamente algunos resultados obtenidos, relativos a la discretización de una variable aleatoria $X$ continua para la cual todos los momentos $\alpha_{\mathrm{k}}=\mathrm{E}\left[\mathrm{X}^{\mathrm{k}}\right]$ existen y son finitos para $\mathrm{k}=0,1,2, \ldots$ 
Sea $f(x)$ la función de densidad de la variable $X$ y sea Sop $(f X)=[a, b]$ (intervalo acotado o no) el soporte de dicha función, entonces en el espacio

$$
L^{2}(a, b, f(x))=\left\{g(x) / \int_{a}^{b}|g(x)|^{2} f(x) d x<\infty\right\}
$$

se puede definir el producto escalar

$$
<g, h>=\int_{a}^{b} g(x) h(x) f(x) d x .
$$

Dicho producto permite generar una sucesión de polinomios ortogonales en $(a, b)\left\{P_{n}(x)\right\} n \geq 0$ relativos a $f_{X}(x)$. Teniéndose el siguiente resultado:

I) "Sean $t_{n i} \epsilon(a, b) ; i=1, \ldots, n$ las $n$ raíces del polinomio ortogonal de grado $n, P_{n}(x)$ relativo a $f_{X}(x)$. Sea el sistema de incógnitas $p_{n i} ; i=1, \ldots, n$

$$
\sum_{i=1}^{n} P_{k}\left(t_{n i}\right) P_{n i}=\left\{\begin{array}{ll}
1 & \text { si } k=0 \\
0 & \text { si } k=1,2, \ldots, n-1
\end{array}\right\}
$$

Entonces (2.2) tiene solución única y se verifica que

$$
p_{n i}>0 ; i=1, \ldots, n \text { y } \quad \sum_{i=1}^{n} p_{n i}=1
$$

pudiendo definirse la variable aleatoria discreta $X_{n}$ de forma que Prob $\left\{X_{n}=t_{n i}\right\}=p_{n i}, \quad, i=1, \ldots, n$ resultando

$$
\alpha_{k}^{*}(n)=E\left[X_{n}^{k}\right]=\alpha_{k} ; k=0,1, \ldots, 2 n-1
$$

no siendo posible la igualdad anterior para $k=0,1, \ldots, 2 n$.

Reciprocamente si $t_{n i}, p_{n i} ; i=1, \ldots, n$ son tales que $\alpha^{*}{ }_{k}(n)=\alpha_{k} ; k=0,1, \ldots, 2 n-1$. Entonces $t_{n i}$ son las raices del polinomio $P_{n}(x)$ relativo a $f(x)$ y los valores $p_{n i}$ verifican (2.2)".

La demostración de este resultado se basa en las propiedades de la cuadratura de Gauss (v. [13] y [16]).

Los resultados de este teorema pueden esquematizarse tal y como se indica en la Fig. 2.3, en la cual para $X$ y $X_{n}$ se dan, simultáneamente y estableciendo un cierto paralelismo, las expresiones de la función de densidad (para $X_{n}$ en términos de la "delta de Dirac"), de los momentos respecto al origen y de la función de distribución. Asimismo también se indica que los valores $t_{n i l} p_{n}$ i quedan caracterizados por la condición de igualdad de momentos hasta el orden $2 n-1$.
Véase que de esta forma para cada $\mathrm{n} \epsilon \mathrm{IN}$ queda definida $X_{n}$ y que por tanto puede construirse una sucesión $\left\{X_{n}\right\} n \geq 1$ asociada a $X$. Debe observarse por otro lado que $\left\{X_{n}\right\}_{n} \geq 1$ se basa sólo en los momentos $\alpha_{k} ; k=$ $=0,1, \ldots$ y no en la distribución dada por $f X$, es decir $X$ puede no quedar unívocamente determinada por sus momentos como le sucede a las variables LogarítmicoNormales (v. [13]). Sin embargo, cuando X queda unívocamente determinada por sus momentos (por ejemplo cuando $X$ es acotada), la convergencia de momentos de $X_{n}$ a $X$ esto es para cada $k, \alpha^{*}{ }_{k}(n) \rightarrow \alpha_{k}$ garantiza la convergencia en distribución de $\left\{X_{n}\right\}_{n} \geq 1$ a $X$ (v. [11] y [12]) teniéndose:

II) "Sea X una variable aleatoria continua (acotada o no) cuya función de distribución FX está univocamente determinada por sus momentos $\left\{\alpha_{k}\right\} k \geq 0$ por hipótesis existentes y finitos. Entonces los momentos de $X_{n}$ convergen a los momentos de $X$ y la sucesión $\left\{X_{n}\right\}$ converge en distribución a X".

La demostración de este resultado se basa en el hecho de que $X_{n}$ reproduce los momentos de $X$ hasta el de orden $2 n-1$.

También para el caso de varias variables aleatorias independientes o reducibles a independientes, puede extenderse el resultado anterior.

Sea una variable aleatoria multidimensional o vector aleatorio $\mathrm{Xt}^{\mathrm{t}}=\left(\mathrm{X}_{1}, \mathrm{X}_{2}, \ldots, \mathrm{X}_{\mathrm{q}}\right)$ cuya función de distribución conjunta:

$$
F_{X}\left(x_{1}, x_{2}, \ldots x_{q}\right)=\operatorname{Prob}\left\{x_{1} \leq x_{1}, x_{2} \leq x_{2}, \ldots, x_{q} \leq x_{q}\right\}
$$

sea absolutamente continua, es decir, que existe una función de densidad conjunta $\mathrm{fx}_{\mathrm{X}}\left(\mathrm{x}_{1}, \mathrm{x}_{2}, \ldots, \mathrm{x}_{\mathrm{q}}\right)$ tal que:

$$
F_{X}\left(x_{1}, \ldots, x_{q}\right)=\int_{-\infty}^{x_{1}} \ldots \int_{-\infty}^{x_{q}} f\left(y_{1}, \ldots, y_{q}\right) d y_{1} \ldots d y_{q}
$$

Consideremos aqui el caso en que $\mathrm{X}_{1}, \mathrm{X}_{2}, \ldots, \mathrm{X}_{\mathrm{q}}$ son independientes, es decir:

$$
F_{X}\left(x_{1}, x_{2}, \ldots, x_{q}\right)=f_{X_{1}}\left(x_{1}\right) f_{x_{2}}\left(x_{2}\right) \ldots f_{x_{q}}\left(x_{q}\right)
$$

De esta forma a la variable aleatoria q-dimensional $\mathrm{X}^{\mathrm{t}}=\left(\mathrm{X}_{1}, \mathrm{X}_{2}, \ldots, \mathrm{X}_{\mathrm{q}}\right)$ podremos asociarle una variable aleatoria discreta

$$
x_{n}^{t}=\left(x_{n_{1}}^{(1)}, x_{n_{2}}^{(2)}, \ldots, x_{n_{q}}^{(q)}\right) \text { con } n=\left(n_{1}, \ldots, n_{q}\right)
$$

donde

$$
X_{n_{r}}^{(r)}=\left(\begin{array}{ccc}
P_{n_{r}}^{(r)} & \ldots & P_{\eta_{\eta}}^{(r)} \\
t_{n_{r} 1}^{(r)} & \ldots & t_{\eta}^{(r)} \\
n_{r}
\end{array}\right), r=1, \ldots, q,
$$




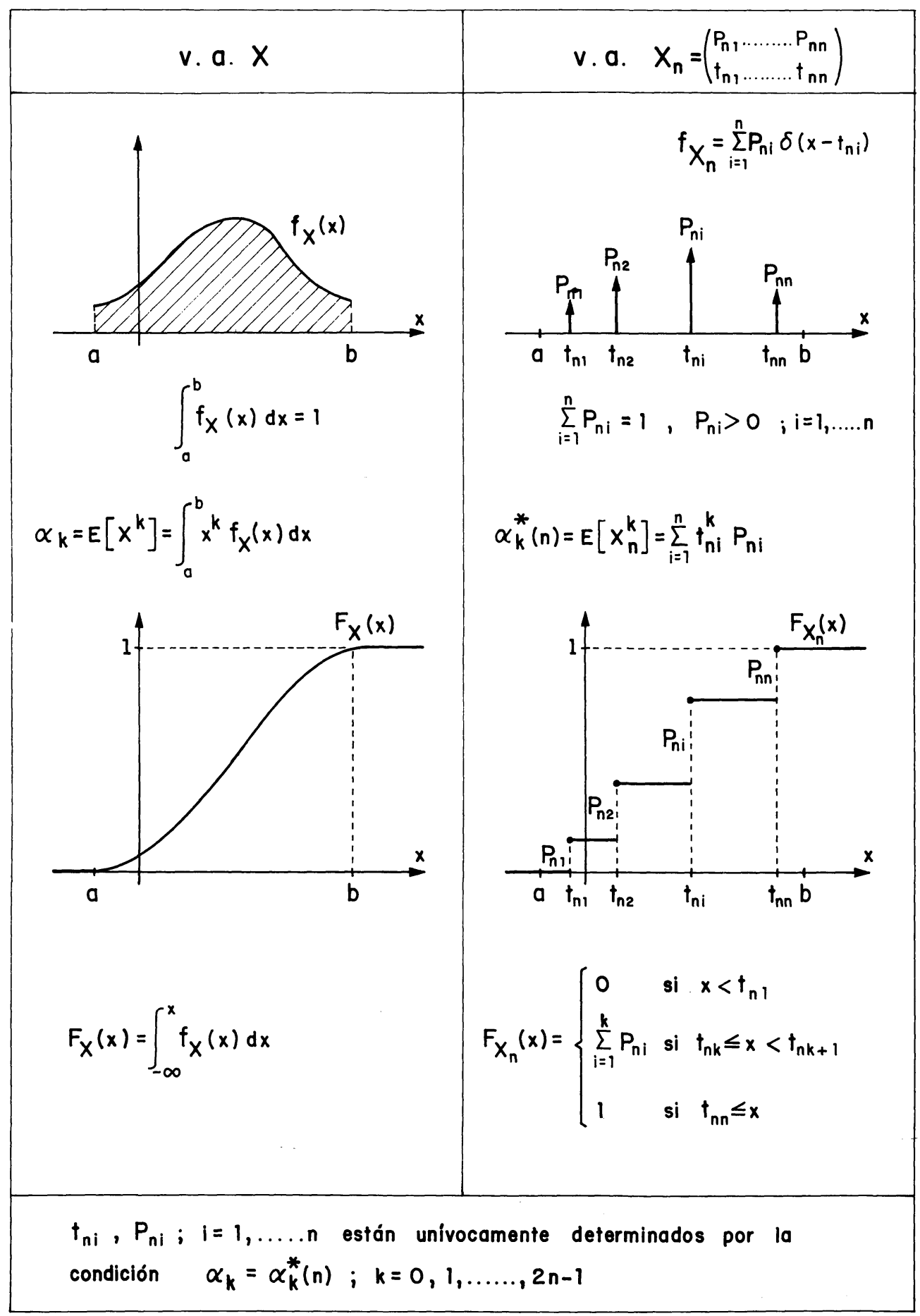

Fig. 2.3

coincide co $X_{r}$ en momentos hasta el orden $2 n_{r}-1$. Teniendo en cuenta la independencia

$$
\begin{gathered}
\operatorname{Prob}\left\{x_{n_{1}}^{(1)}=t_{n_{1} i_{1}}^{(1)} \ldots, x_{n_{q}}^{(q)}=t_{n_{q} i_{q}}^{(q)}\right\}= \\
p_{n_{1} i_{1}}^{(1)} \ldots p_{n_{q} i_{q}}^{(q)} ; i_{1}=1, \ldots, n_{1} ; \ldots ; i_{q}=1, \ldots, n_{q}
\end{gathered}
$$$$
X_{n}^{t}=\left(\begin{array}{c}
R_{n_{1} i_{1}}^{(1)} R_{n_{2} i_{2}}^{(2)} \cdots \cdots \cdot R_{n_{q} i_{q}}^{(q)} \\
\left(t_{n_{1} i_{1}}^{(1)}, t_{n_{2} i_{2}}^{(2)} \ldots \ldots \ldots t_{n_{q} i_{q}}^{(q)}\right)_{1 \times q}
\end{array}\right)_{2 \times\left(n_{1} \times n_{2} \times . \times n_{q}\right)}
$$

de esta manera $X_{n}$ puede expresarse en notación matricial como:

$$
i_{1}=1, \ldots, n_{1} ; i_{2}=1, \ldots, n_{2} ; \ldots ; i_{q}=1, \ldots, n_{q}
$$


Sean

$$
\alpha_{k_{1} k_{2} \ldots k_{q}}=E\left[x_{1}^{k_{1}} x_{2}^{k_{2}} \ldots x_{q}^{k_{q}}\right]
$$

los momentos de orden $\mathrm{k}_{1}, \mathrm{k}_{2}, \ldots, \mathrm{k}_{\mathrm{q}}$ del vector aleatorio $X$ los cuales supondremos existentes $y$ finitos para todo $\mathrm{k}_{1} \geq 0, \mathrm{k}_{2} \geq 0, \ldots, \mathrm{k}_{\mathrm{q}} \geq 0$, y sean asimismo:

$$
\begin{gathered}
\alpha_{k_{1} k_{2} \ldots k_{q}}^{*}\left(n_{1}, n_{2}, \ldots, n_{q}\right)=E\left[x_{n_{1}}^{(1) k_{1}} x_{n_{2}}^{(2) k_{2}} \ldots x_{n_{q}}^{(q) k q}\right]= \\
=\sum_{i_{1}=1}^{n_{1}} \sum_{i_{2}=1}^{n_{2}} \ldots \sum_{i_{q}=1}^{n_{q}}\left(t_{n_{1} i_{1}}^{(1)}\right)^{k_{1}}\left(t_{n_{2} i_{2}}^{(2)}\right)^{k_{2}} \ldots \\
\cdots\left(t_{n_{q} i_{q}}^{(q)}\right)^{k_{q}} p_{n_{1}}^{(1)} p_{n_{2} i_{2}}^{(2)} \ldots p_{n_{q} i_{q}}^{(q)}
\end{gathered}
$$

los momentos de orden $\mathrm{k}_{1}, \mathrm{k}_{2}, \ldots, \mathrm{k}_{\mathrm{q}}$ del vector aleatorio $X_{n}$.

Un resultado inmediato que se obtiene como consecuencia de la independencia es:

III) "Sean

$$
\alpha_{k_{1} k_{2} \ldots k_{q}} \text { y } \alpha_{k_{1} k_{2} \ldots k_{q}}^{*}\left(n_{1}, n_{2}, \ldots, n_{q}\right)
$$

los momentos anteriormente definidos. Se verifica que:

$$
\alpha_{k_{1} k_{2} \ldots k_{q}}=\alpha_{k_{1} k_{2} \ldots k_{q}}^{*}\left(n_{1}, n_{2}, \ldots, n_{q}\right)
$$

para cada

$$
k_{1}=0,1, \ldots, 2 n_{1}-1 ; k_{2}=0,1, \ldots, 2 n_{2}-1, \ldots, k_{q}=0,1, \ldots, 2 n_{q}-1 " .
$$

$Y$ análogamente al caso unidimensional también se tiene:

IV) "Si las distribuciones marginales quedan unívocamente determinadas por las sucesiones de momentos entonces la sucesión de vectores aleatorios $X_{n}$ converge en distribución a X".

En [13] se han extendido estos resultados al caso de variables normales q-dimensional cuyas componentes son estocásticamente dependientes.

A continuación pasamos a definir la distribución discreta asociada a una variable aleatoria $Y$ que depende de otras variables aleatorias independientes $X_{1}$, $X_{2}, \ldots, X_{q}$ a través de una función medible $g$, esto es: $Y=g\left(X_{1}, X_{2}, \ldots, X_{q}\right)$.
Teniendo en cuenta que $X_{n}$ es discreta, definimos a $Y_{n}$ variable aleatoria discreta asociada a $Y$ como $Y_{n}=$ $=g\left(X_{n}\right) \circ$ más explícitamente:

$$
Y_{n_{1} n_{2} \ldots n_{q}}=g\left(X_{n_{1}}^{(1)}, X_{n_{2}}^{(2)}, \ldots, X_{n_{q}}^{(q)}\right)
$$

Esta variable aleatoria discreta unidimensional $Y_{n}$, puede expresarse con el simbolismo matricial en la forma:

$Y_{n_{1} n_{2} \ldots n_{q}}=\left(\begin{array}{cccc}p_{n_{1} i_{1}}^{(1)} & p_{n_{2} i_{2}}^{(2)} \ldots & p_{n_{q} i_{q}}^{(q)} \\ g\left(t_{n_{1} i_{1}}^{(1)}, t_{n_{2} i_{2}}^{(2)}\right. & \ldots, & \left.t_{n_{q} i_{q}}^{(q)}\right)\end{array}\right) 2 \times\left(n_{1} \times n_{2} \times \ldots \times n_{q}\right)$

asimismo la expresión anterior deberá ponerse más propiamente como:

$$
Y_{n_{1} n_{2} \ldots n_{q}}=\left(\begin{array}{cccc}
p_{1} & p_{2} & \cdots & p_{m} \\
y_{1} & y_{2} & \cdots & y_{m}
\end{array}\right)
$$

donde $\left\{\mathrm{y}_{1}, \mathrm{y}_{2}, \ldots, \mathrm{y}_{\mathrm{m}}\right\}$ es el conjunto (ordenado en sentido creciente) de las imágenes obtenidas mediante $\mathrm{g}$ del conjunto de puntos

$$
\left\{\left(t_{n_{1} i_{1}}^{(1)}, t_{n_{2} i_{2}}^{(2)}, \ldots, t_{n_{q} i_{q}}^{(q)}\right) ; i_{1}=1, \ldots, n_{1} ; i_{2}=1, \ldots, n_{2} ; \ldots ; i_{q}=1, \ldots, n_{q}\right\}
$$

y

$$
P_{j}=\sum P_{n_{1} i_{1}}^{(1)} P_{n_{2} i_{2}}^{(2)} \ldots P_{n_{q} i_{q}}^{(q)}
$$

extendiendo el sumatorio al conjunto de puntos tales que

$$
g\left(t_{n_{1} i_{1}}^{(1)}, t_{n_{2} i_{2}}^{(2)}, \ldots, t_{n_{q} i_{q}}^{(q)}\right)=y_{j}
$$

con $\mathrm{j}=1, \ldots, \mathrm{m}$ donde $m \leq n_{1} \times n_{2} \times \ldots \times n_{q}$.

Obsérvese que la construcción de la función de distribución $F Y_{n}(y)$ es una operación inmediata a partir de (2.3).

El análisis de la convergencia de momentos y de la convergencia en distribución es una cuestión delicada cuando se trata del caso de variables aleatorias no acotadas. Sin embargo para el caso de variables acotadas hemos demostrado (v. [13]) el siguiente resultado:

V) "Sean $X_{1}, X_{2}, \ldots, X_{q} v$. a's continuas acotadas e independientes y sea $Y=g\left(X_{1}, X_{2}, \ldots, X_{q}\right)$ con $g$ continua, una $v$. a que depende de $X_{1}, X_{2}, \ldots, X_{q}$, .Sean

$$
\beta_{k}=E\left[r^{k}\right] \text { y } \beta_{k}^{*}\left(n_{1}, n_{2}, \ldots, n_{q}\right)=E\left[r_{n_{1} n_{2} \ldots n_{q}}^{k}\right]
$$

los momentos de $Y$ e $Y_{n_{1}} n_{2} \ldots n_{4}$ respectivamente. 
Entonces para cada $\mathrm{k} \in \mathrm{IN}$ se tiene:

$$
\begin{aligned}
& \lim _{n_{1} \beta^{*}{ }^{k}}\left(n_{1}, n_{2}, n_{q}\right)=\beta_{k} \\
& \vdots \\
& n_{q} \rightarrow \infty
\end{aligned}
$$

$y\left\{Y_{n_{1} n_{2} \ldots n_{q}}\right\}$ converge en distribución a $Y^{\prime \prime}$.

\section{Descripción del método}

Como resumen de todo lo anterior se va ha exponer el procedimiento desarrollado para aproximar los momentos, función de distribución, etc., de una variable aleatoria $Y$ que es función de otras variables aleatorias $X_{1}, X_{2}, \ldots, X_{q}$ independientes.

Por otra parte, como se indicó anteriormente la función $g$ que relaciona a $Y$ con $X_{i} ; i=1, \ldots$, q puede estar dada explícitamente, implícitamente o venir definida mediante un problema de valor inicial de contorno, etc.

Asimismo, fue precisamente la resolución de problemas de autovalores estocásticos, y dentro de ellos los de pandeo estocástico lo que nos condujo definitivamente al desarrollo del método que en este trabajo exponemos.

Las etapas básicas de dicho método pueden esquematizarse tal y como se indica en la Fig. 3.1.

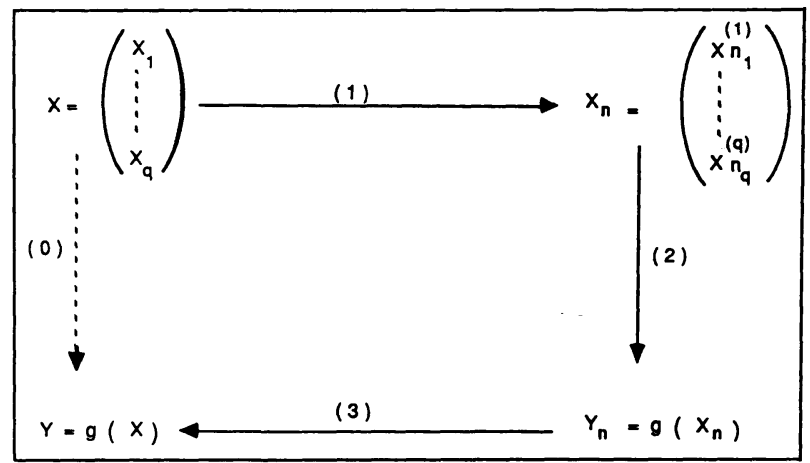

Fig. 3.1

donde los pasos (1), (2), y (3) equivalen a:

(1) Fijar los valores $n_{1}, n_{2}, \ldots, n_{q} \in \mathbb{I N}$ para las respectivas variables $X_{1}, X_{2}, \ldots, X_{q}$. Construir para cada $r=$ $=1,2, \ldots, q$ la variable aleatoria discreta finita.

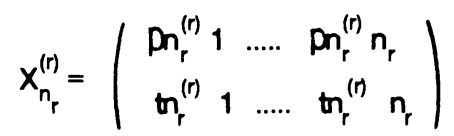

asociada a Xr que coincide con ella en momentos hasta el de orden $2 n_{r-1}$.
Finalmente determinar la variable discreta q-dimensional

$$
x_{n}^{t}=\left(X n_{1}^{(1)}, X n_{2}^{(2)}, \ldots, X_{q}^{(q)}\right)
$$

asociada a la variable vectorial $q$-dimensional

$$
x^{t}=\left(x_{1}, x_{2}, \ldots, x_{q}\right)
$$

donde:

$$
\begin{aligned}
& x_{n}^{t}=\left(X n_{1}^{(1)}, x_{n 2}^{(2)}, \ldots, X_{q}^{(q)}\right)=
\end{aligned}
$$

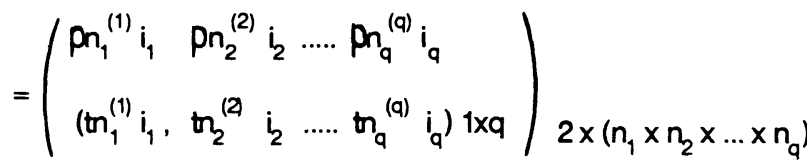

$$
\begin{aligned}
& i_{1}=1, \ldots, n_{1} ; i_{2}=1, \ldots, n_{2} ; i_{q}=1, \ldots, n_{q}
\end{aligned}
$$

El cálculo de los puntos de discretizácición

$$
t_{n_{1} i}^{(r)} ; i=1, \ldots, n_{r}
$$

y las probabilidades asociadas

$$
p_{n_{r} i}^{(r)} ; i=1, \ldots, n_{r}
$$

una vez fijado $n_{r}$, puede efectuarse por diversos procedimientos, sin embargo los más recomendables desde el punto de vista numérico son aquellos que se basan en el conocimiento de la relación de recurrencia que satisfacen los polinomios ortogonales generados a partir de las distribuciones de $X_{r} ; r=1, \ldots, q$. En concreto uno de los más eficaces y por ello muy utilizado también en la construcción de fórmulas de cuadratura gaussianas, es aquél en el cual los valores

$$
t_{n_{1},}^{(r)} \text { y } p_{n_{r} i}^{(n)} ; i=1, \ldots, n_{r}
$$

se obtienen como elementos característicos de una matriz llamada tridiagonal de Jacobi construida a partir de los coeficientes de la relación de recurrencia ( $v$. [9] y [13]).

Por otra parte, algunas distribuciones de variables aleatorias notables, tienen unas funciones de densidad que son análogas a las funciones peso de los polinomios ortogonales clásicos: Jacobi, Laguerre y Hermite. Asimismo, dichos polinomios ortogonales clásicos poseen unas relaciones de recurrencia cuyos coeficientes se conocen en función de $n$ y mediante expresiones sencillas. De esta forma el cálculo de raíces y números de Christoffel (constantes de ponderación de una fórmula de cuadratura, en nuestro caso probabili- 
dades asociadas) para estos polinomios puede llevarse a cabo con gran exactitud. En este sentido, uno de los manuales que contiene varios programas de cálculo y tablas que dan los ceros y números de Christoffel para una amplia gama de funciones peso incluídas las de los polinomios clásicos, es el de Stroud y Secrest [17].

En [13] exponemos la relación entre los valores $t_{n i}$, $p_{n i}$; $i=1, \ldots, n$ de la variable aleatoria $X_{n}$ asociada a la variable $\mathrm{X}$ en los casos de variable Uniforme, Normal, Exponencial, Gamma y Beta y los ceros y números Christoffel de los respectivos polinomios ortogonales clásicos. También en dicha referencia se indica la forma de proceder en la construcción de las variables discretas cuando las variables de partida poseen distribuciones no clásicas.

(2) Obtener la variable aleatoria discreta $Y_{n} 0$ con notación más explícita $Y n_{1} \ldots n_{q}$ asociada a

$$
Y=g\left(X_{1}, X_{2}, \ldots, X_{q}\right)
$$

donde $Y n_{1} n_{2}, \ldots n_{q}=g\left(X n_{1}^{(1)}, X n_{2}^{(2)}, \ldots, X n_{q}^{(q)}\right)=$

$=\left(\begin{array}{cccc}p n_{1}^{(1)} i_{1} & p n_{2}^{(2)} & i_{2} \ldots & p n_{q}^{(q)} i_{q} \\ g\left(m n_{1}^{(1)} i_{1}\right. & , & m_{2}^{(2)} i_{2}, \ldots, & \left.m_{q}^{(q)} i_{q}\right) 1 \times q\end{array}\right)_{2 \times\left(n_{1} \times n_{2} \times \ldots \times n_{q}\right)}$

$\operatorname{con} i_{1}=1, \ldots, n_{1} ; i_{2}=1, \ldots, n_{2} ; i_{q}=1, \ldots, n_{q}$.

Tal y como se indicó, dicha variable discreta, una vez ordenadas las imágenes y sumadas las probabilidades respectivas, se pondrá en la forma:

$$
Y n_{1} n_{2} \ldots n_{a}=\left(\begin{array}{cccc}
p_{1} & p_{2} & \cdots . . & p_{m} \\
y_{1} & y_{2} & \cdots & y_{m}
\end{array}\right)
$$

Por otro lado, en el caso de que g esté dada explícitamente, el cálculo de $Y n_{1} n_{2} \ldots n_{q}$ se reducirá a la simple evaluación de $g$ en $n_{1} \times n_{2} \times \ldots . . \times n_{q}$ puntos.

Asimismo, cuando la relación entre $Y$ y $X_{i} ; i=1, \ldots, q$ sea implícita, la determinación de la variable discreta asociada $Y n_{1} n_{2} \ldots n_{q}$ implicará la resolución de $n_{1} \times n_{2}$ $x n_{q}$ ecuaciones algebraicas $o$ trascendentes de tipo determinista.

Análogamente, cuando g esté definida mediante un problema estocástico (de valor inicial, de contorno, etc) la determinación de la citada variable discreta requerirá la resolución numérica de $n_{1} \times n_{2} \times \ldots \times n_{q}$ problemas deterministas.

(3) Estimar los momentos de $Y$ a partir de los correspondientes de $\mathrm{Yn}_{1} \mathrm{n}_{2} \ldots \mathrm{n}_{\mathrm{q}}$. Asimismo construir también una función de distribución aproximada para la variable aleatoria $Y$ tomando como referencia la función de distribución de $Y_{1} n_{2} \ldots n_{q}$. En este sentido puede tomarse a la propia distribución de la variable discreta $\mathrm{Yn}_{1} \mathrm{n}_{2} \ldots \mathrm{n}_{\mathrm{g}}$ como función de distribución aproximada en virtud de la convergencia en distribución de la sucesión $\left\{\mathrm{n}_{1} \mathrm{n}_{2} \ldots \mathrm{n}_{\mathrm{q}}\right\}$ a Y. No obstante puede interesar en ciertos casos ajustar una distribución clásica a partir de la información contenida en la distribución aproximada (por ejemplo a partir de los momentos).

También se ha de indicar que en aquellos casos de convergencia (por ejemplo g continua con $x_{i} ; 1, \ldots, q$ acotadas) el método expuesto permite aproximar tanto como se desea las características estocásticas de $Y$ a partir de las de $Y n_{1} n_{2} \ldots n_{q}$ aumentando el número de puntos de discretización.

\section{Aplicaciones}

La aplicación del método de discretización propuesto a diferentes supuestos prácticos, en los que son conocidas las distribuciones de probabilidad de las variables aleatorias independientes que intervienen en el problema y la existencia de sus momentos de cualquier orden, pone de manifiesto que es superior en muchas situaciones a otros métodos existentes, en aspectos tales como la sencillez de los cálculos analíticos necesarios o la bondad de las aproximaciones para un esfuerzo de cálculo similar.

Seguidamente se exponen dos ejemplos, en el primero la función g está dada explícitamente. En el segundo dicha función viene definida mediante un problema de autovalores estocásticos.

\section{Ejemplo 1}

Se desarrolla aqui una aplicación del método propuesto a un problema particular de Mecánica de Suelos.

Concretamente nos proponemos estimar los primeros momentos de la resistencia al esfuerzo cortante $\tau$ de un suelo mediante la fórmula de Coulomb:

$$
\tau=C+\sigma \operatorname{tg} \varnothing
$$

suponiendo que la cohesión C, la tensión normal $\sigma$ y el ángulo de rozamiento $\phi$ son variables aleatorias independientes, con unas distribuciones de probabilidad conocidas.

Para este caso se ha supuesto que las variables de partida $\mathrm{C}, \sigma$ y $\phi$ tienen distribución uniforme en los intervalos siguientes: $\mathrm{C}\left(\mathrm{kg} / \mathrm{cm} .{ }^{2}\right)$ en $[4,24] \sigma\left(\mathrm{kg} / \mathrm{cm} .{ }^{2}\right)$ en $[9,21]$ y $\phi$ (grados) en $[11,26]$. 
Los momentos de $\tau$ se estiman a partir de los correspondientes de $\tau n_{1} n_{2} n_{3}$ variable aleatoria discreta definida como:

$$
\tau n_{1} n_{2} n_{3}=g\left(C n_{1}, \sigma n_{2}, ø n_{3}\right)=C n_{1}+\sigma n_{2} \operatorname{tg} ø n_{3}
$$

donde $\mathrm{Cn}_{1}$, on $\mathrm{n}_{2}$ y $\phi \mathrm{n}_{3}$ son variables aleatorias discretas asociadas respectivamente a $\mathrm{C}, \sigma, \mathrm{y} \phi$.

Dichas variables se construyen a partir de los ceros y números de Christoffel de los polinomios de Legendre, en la forma que a continuación se indica (v. [13]):

$$
X_{n}=\left\{\begin{array}{l}
p_{n i}=A_{n i} / 2 \quad \text { (probabilidades) } \\
t_{n i}=\frac{b-a}{2} Z_{n i}+(a+b) / 2 \\
i=1, \ldots, n
\end{array}\right.
$$

donde $Z_{n i}, A_{n i} ; i=1, \ldots, n$ son respectivamente los ceros y números de Christoffel de los polinomios de Legendre de grado $n$ (los relativos a la función peso $w(x)=1$ en $-1 \leq x \leq 1)$ y $X_{n}$ en la variable aleatoria discreta asociada a la variable $X$ uniforme en $[a, b]$.

En Stroud y Secrest [17] se encuentran tabulados $Z_{n i}$, $A_{n i} ; i=1, \ldots, n$ desde $n=2$ hasta $n=512$ con una precisión de treinta dígitos decimales.

En este caso las variables $\mathrm{Cn}_{1}, \sigma \mathrm{n}_{2}$ y $\phi \mathrm{n}_{3}$ son:

$$
C n_{1}=\left\{\begin{array}{l}
p n_{1}^{(1)} i=A n_{1} i / 2 \\
n_{1}^{(1)} i=\frac{24-4}{2} Z n_{1} i+\frac{24+4}{2} \\
i=1,2, \ldots, n_{1}
\end{array}\right.
$$

$$
\begin{aligned}
& \sigma n_{2}=\left\{\begin{array}{l}
\mathrm{pn}_{2}^{(2)} \mathrm{i}=A n_{2} \mathrm{i} 2 \\
\mathrm{t}{ }_{2}^{(2)} i=\frac{21-9}{2} Z n_{2} i+\frac{9+21}{2} \\
i=1,2, \ldots, n_{2}
\end{array}\right. \\
& \sigma n_{3}=\left\{\begin{array}{l}
\mathrm{pn}_{3}^{(3)} i=A n_{3} \mathrm{i} 2 \\
\mathrm{t} n_{3}^{(3)} i=\frac{26-11}{2} \mathrm{Zn}_{3} i+\frac{11+26}{2} \\
i=1,2, \ldots, n_{3}
\end{array}\right.
\end{aligned}
$$

En la Tabla 4.1 se dan los momentos $\beta^{*}{ }^{*}\left(n_{1} n_{2} n_{3}\right)=$ $=E\left[\tau^{k} n_{1} n_{2} n_{3}\right]$ de orden inferior a cinco, en función del grado de discretización. En este caso las variables de partida son discretizadas en el mismo número de puntos, es decir $n_{1}=n_{2}=n_{3}=n$.

Los momentos son simbolizados en la Tabla 4.1 por $\beta^{*} k(n)$.

Los resultados obtenidos ponen de manifiesto la convergencia de los momentos de la sucesión de variables aleatorias $\left\{\pi_{1} n_{2} n_{3}\right\}$ a los momentos del mismo orden de la variable aleatoria $\tau$. Obsérvese que incluso para valores de $n$ pequeños $(n=2,3$, etc.) las aproximaciones de los momentos a los valores límites resultan ser muy aceptables.

En las figuras 4.1 a 4.6 pueden observarse las gráficas de las funciones de distribución de $\tau, n, n, n$ para $n=5,6,7,8,9$ y 10 , en donde asimismo queda patente la convergencia en distribución de $\left\{\tau_{n}, n, n\right\}$ a $\tau$.

Nótese que el recorrido de $\tau$ es el intervalo

$$
[5.749,34.242] \text { pues }
$$

$\tau_{\text {mín. }}=4+9 \operatorname{tg} 11=5.749, \tau_{\text {máx. }}=24+21 \operatorname{tg} 26=34.242$

\begin{tabular}{|c|c|c|c|c|}
\hline $\mathbf{n}$ & Media $=\beta^{*}{ }^{*}(\mathbf{n})$ & $\begin{array}{c}\text { Varianza }= \\
=\beta_{2}{ }^{*}(\mathbf{n}) \cdot \beta_{1}{ }^{* 2}(\mathbf{n})\end{array}$ & $\beta_{3}{ }^{*}(\mathbf{n})$ & $\beta_{4}^{*}(\mathbf{n})$ \\
\hline 1 & 19.0189298 & 0.00000000 & 6879.52140 & 130841.135 \\
\hline 2 & 19.0509474 & 36.3762305 & 8995.91047 & 212873.814 \\
\hline 3 & 19.0510621 & 36.3840609 & 8996.65082 & 213802.577 \\
\hline 4 & 19.0510624 & 36.3840900 & 8996.65414 & 213802.783 \\
\hline 5 & 19.0510624 & 36.3840935 & 8996.65415 & 213802.784 \\
\hline
\end{tabular}




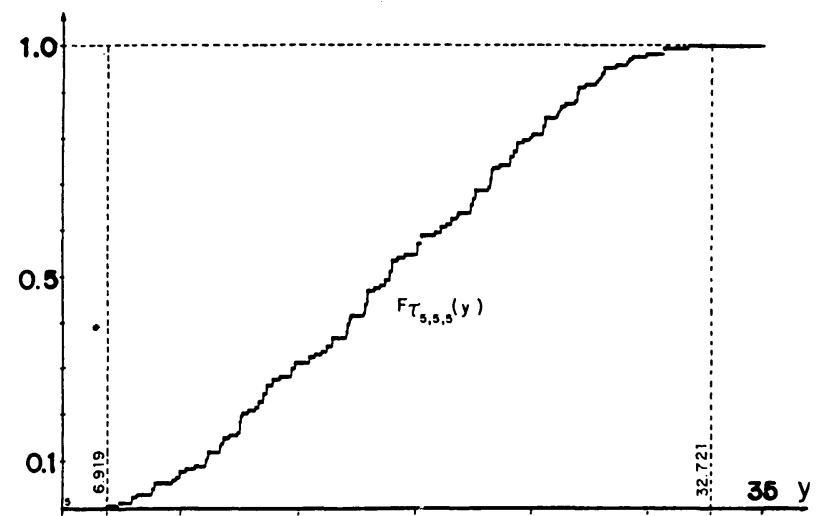

Fig. 4.1

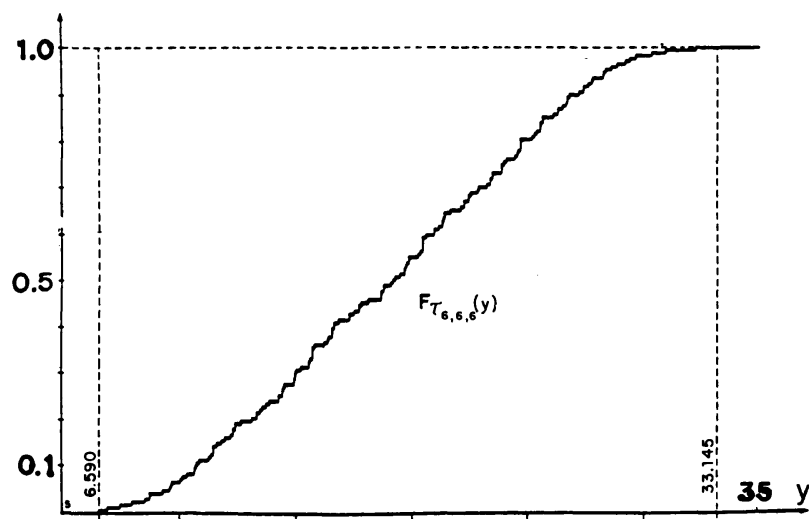

Fig. 4.2

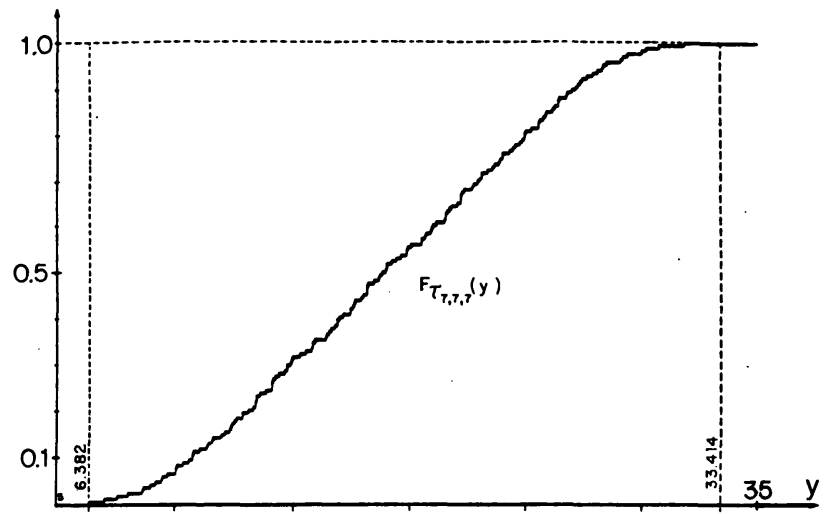

Fig. 4.3

A continuación se realiza una estimación de los momentos de $\tau$, mediante la simulación de Monte Carlo, con objeto de comparar dichos resultados con los obtenidos por el método propuesto. En la Tabla 4.2 se indican los valores obtenidos en cuatro realizaciones distintas para muestras de tamaño $N=100,1000$ y 10000 respectivamente. Los momentos muestrales son designados por $\beta^{*}$.

A destacar la lenta convergencia de los valores obtenidos hacia los valores límites; en este sentido véase incluso que con muestras de tamaño $N=10000$ no puede ni siquiera darse el primer dígito decimal de la

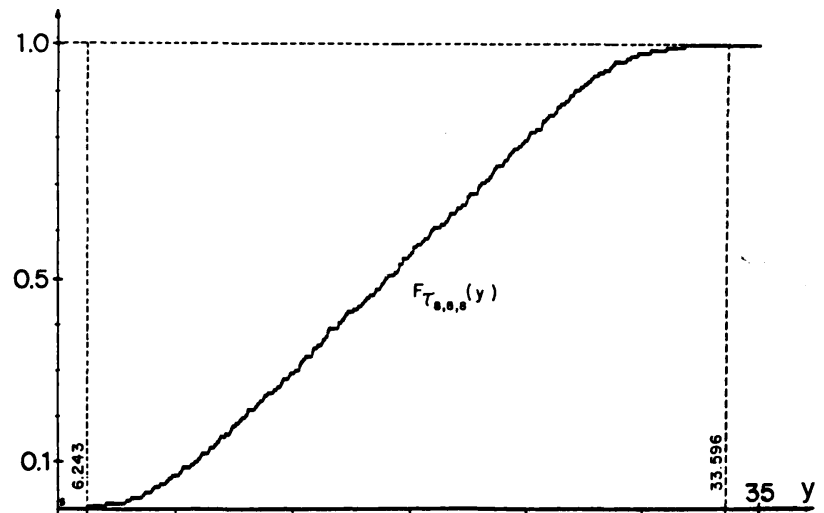

Fig. 4.4

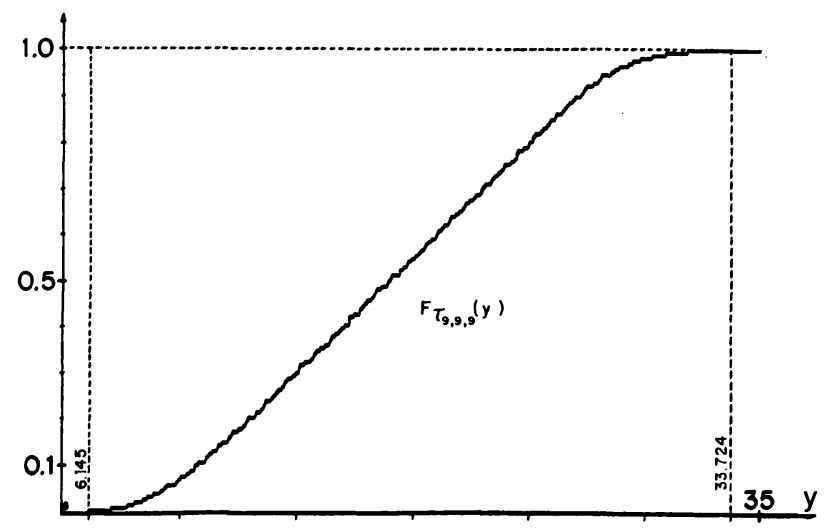

Fig. 4.5

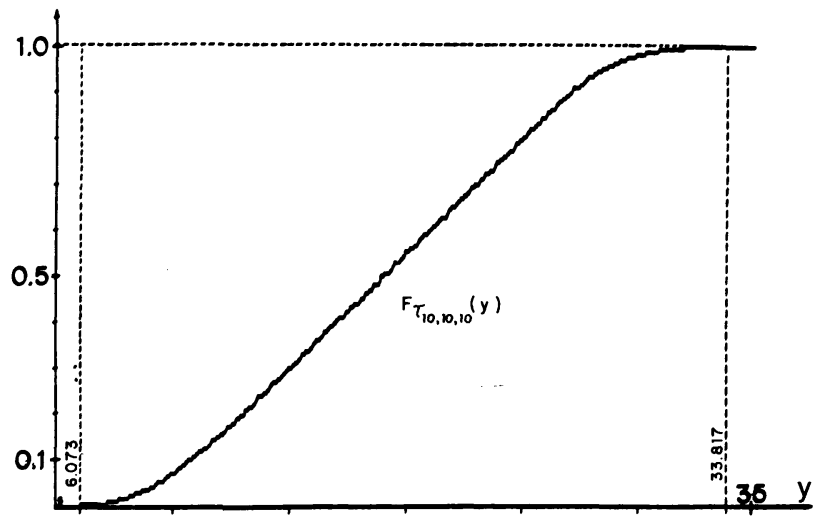

Fig. 4.6

media como cifra segura.

Asimismo, en las Figuras 4.7 a 4.10 pueden observarse las gráficas de distribuciones empíricas de $\tau: \mathrm{F} 125_{\tau}(\mathrm{y})$ y $\mathrm{F} 1000_{\tau}$ (y) construidas para dos realizaciones muestrales de tamaño $N=125$ y 1000 respectivamente. Comparando estas funciones de distribución aproximadas de $\tau$ con sus homólogas (en lo relativo al número de puntos) $F_{\tau 5,5,5}(\tau)$ y $F_{\tau 10,10,10}(y)$ puede ponerse de ma. nifiesto la gran influencia de la realización muestral, en la forma de las mismas, lo cual permite destacar una vez más las posibilidades del método propuesto. 


\begin{tabular}{|c|c|c|c|c|c|}
\hline & $\begin{array}{c}\text { Realización } \\
\text { muestral }\end{array}$ & Media $=\beta_{1}^{*}$ & $\begin{array}{l}\text { Varianza }= \\
=\beta_{2} * \beta_{1} * 2\end{array}$ & $\beta_{3}^{*}$ & $\beta_{4}^{*}$ \\
\hline \multirow{4}{*}{$\begin{array}{l}8 \\
\text { 우 } \\
\text { II } \\
2\end{array}$} & 1 & 18.8006569 & 40.50171100 & 8936.970740 & 214553.904 \\
\hline & 2 & 19.3460691 & 37.46186820 & 9440.919880 & 228975.666 \\
\hline & 3 & 18.9208930 & 33.90288410 & 8691.071330 & 202745.313 \\
\hline & 4 & 18.6872882 & 37.29314360 & 8613.803080 & 202634.655 \\
\hline \multirow{4}{*}{$\begin{array}{l}8 \\
\text { 우 } \\
\text { II } \\
2\end{array}$} & 1 & 19.2908573 & 36.56049930 & 9274.886090 & 221292.656 \\
\hline & 2 & 19.0225843 & 36.2610905 & 8961.183160 & 212979.810 \\
\hline & 3 & 19.1897518 & 36.34325480 & 9165.841190 & 219031.712 \\
\hline & 4 & 19.4993201 & 36.72415860 & 9663.966780 & 231172.409 \\
\hline \multirow{4}{*}{$\begin{array}{l}8 \\
8 \\
8 \\
\text { ㅇ } \\
z\end{array}$} & 1 & 19.0751374 & 35.86118020 & 8993.849590 & 213321.429 \\
\hline & 2 & 19.1253450 & 37.18206150 & 9131.846850 & 218354.911 \\
\hline & 3 & 18.9964487 & 36.71491780 & 8928.499960 & 212035.758 \\
\hline & 4 & 19.1261746 & 36.43465510 & 9086.080700 & 216339.895 \\
\hline
\end{tabular}

Tabla 4.2

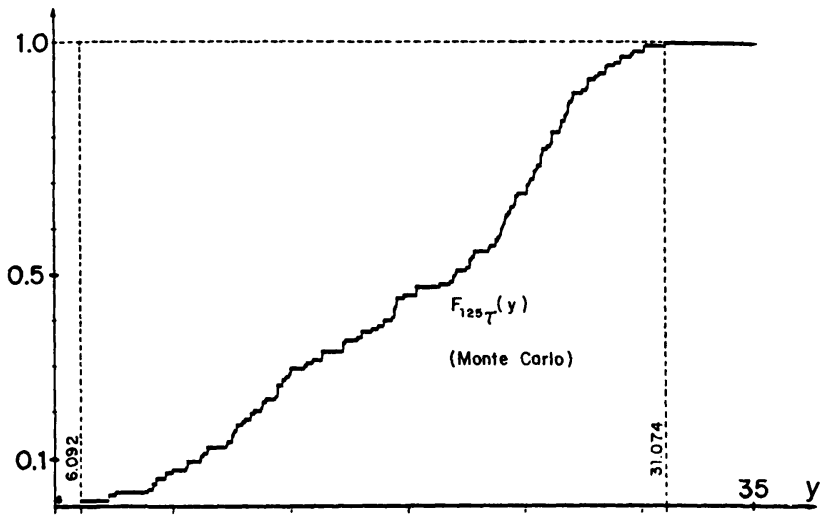

Fig. 4.7

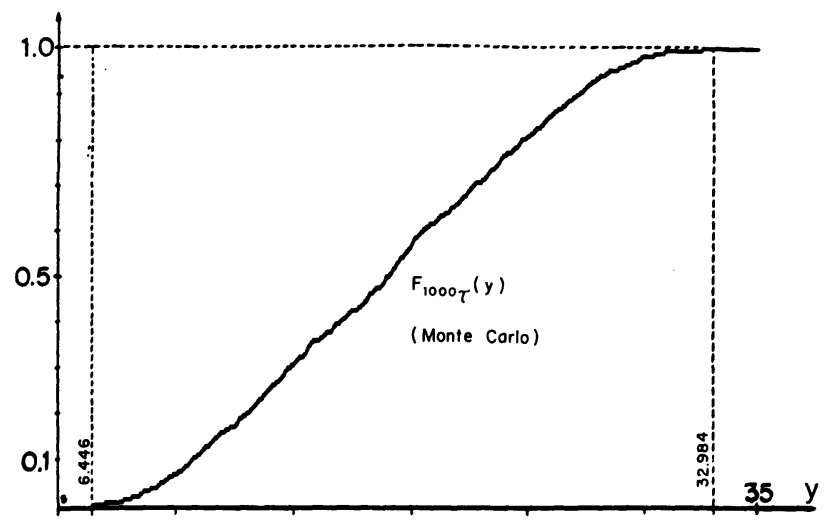

Fig. 4.9

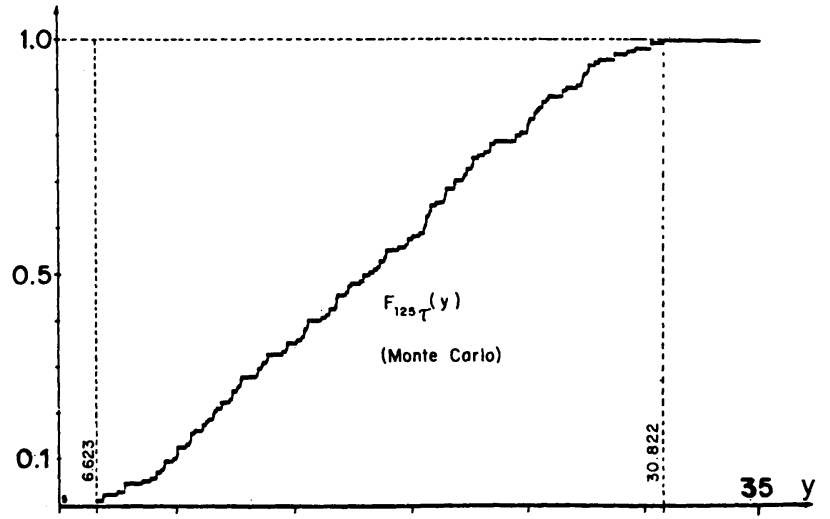

Fig. 4.8

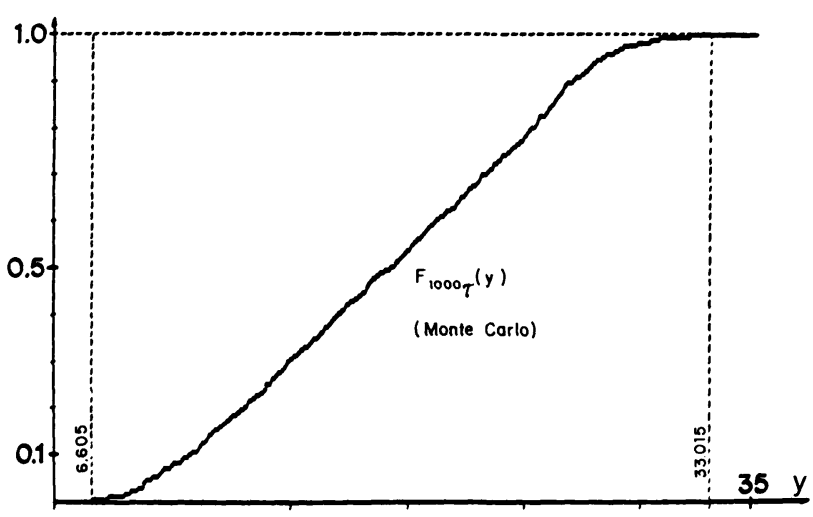

Fig. 4.10 
Calculando estimaciones de la media y la varianza de $\tau$ a partir de las expresiones (4.2) y (4.3), correspondientes al método de Taylor:

$$
\begin{aligned}
& E\left[\equiv g\left(m_{1}, \ldots, m_{q}\right)+\frac{1}{2} \sum_{i=1}^{q}\left(\frac{\partial^{2} g(m)}{\partial x_{i}^{2}}\right) D^{2}\left[X_{i}\right]\right. \\
& D^{2}[Y] \equiv \sum_{i=1}^{q}\left(\frac{\partial g(m)}{\partial x_{i}}\right)^{2} D^{2}\left[X_{i}\right]
\end{aligned}
$$

Llamando

$$
Y=\tau, X_{1}=C, X_{2}=\sigma \text { y } X_{2}=\varnothing(\text { rad. })
$$

Se obtiene:

$$
\begin{aligned}
& \frac{\partial Y}{\partial X_{1}}=1, \frac{\partial Y}{\partial X_{2}}=\operatorname{tg} X_{3}, \frac{\partial Y}{\partial X_{3}}=\frac{X_{2}}{\cos ^{2} X_{3}} \\
& \frac{\partial^{2} Y}{\partial X_{1}^{2}}=0, \frac{\partial^{2} Y}{\partial X_{2}^{2}}=0, \frac{\partial^{2} Y}{\partial X_{3}^{2}}=\frac{2 X_{2} \operatorname{sen} X_{3}}{\cos ^{3} X_{3}}
\end{aligned}
$$

Por otra parte

$$
E\left[X_{1}\right]=14, E\left[X_{2}\right]=15, E\left[X_{3}\right]=0.32288586
$$

$D^{2}\left[X_{1}\right]=33.33333333, D^{2}\left[X_{2}\right]=12, D^{2}\left[X_{3}\right]=5.7115655 \times 10^{-3}$

De esta forma resulta:

$$
\begin{aligned}
E[\tau]=E[Y] \approx E\left[X_{1}\right] & +E\left[X_{2}\right] \operatorname{tg} E\left[X_{3}\right]+\frac{1}{2} \frac{2 E\left[X_{2}\right] \operatorname{sen} E\left[X_{3}\right]}{\cos ^{3} E\left[X_{3}\right]} D^{2}\left[X_{3}\right]= \\
& =19.050814
\end{aligned}
$$$$
D^{2}[\tau]=D^{2}[Y] \times D^{2}\left[X_{1}\right]+\left(\operatorname{tg} E\left[X_{3}\right]\right)^{2} D^{2}\left[X_{2}\right]+\frac{E\left[X_{2}\right]}{\cos ^{2} E\left[X_{3}\right]} D^{2}\left[X_{3}\right]=
$$$$
=36.2657282
$$

Véase que los valores estimados de la media y la varianza de $\tau$ son peores que los obtenidos mediante $\tau 2,2,2$ (v. Tabla 4.1 para $n=2$ ).

Además, la determinación por el método de Taylor de expresiones que aproximen momentos de orden superior a dos e incluso que mejoren los resultados dados por (4.2) y (4.3) para los dos primeros momentos, llega a ser prácticamente inviable, debido a la complejidad de las fórmulas que resultan.

Esta limitación del método anterior, contrasta en gran medida con la sencillez de aplicación de la técnica propuesta.

\section{Ejemplo 2}

Desarrollamos aquí una aplicación del método propuesto al estudio de las vibraciones de un hilo elástico con un extremo fijo en $x=0 y$ el otro apoyado elásticamente en $x=1$. (Fig. 4.11) con una constante $k$ del apoyo tal, que debido a incertidumbres de diversa índole se modeliza como variable aleatoria $\mathrm{k}(\omega)$. Asi los autovalores y autofunciones se obtendrán resolviendo el problema de autovalores estocásticos:

$$
\left\{\begin{array}{l}
y^{\prime \prime}(x)+\lambda y(x)=0 \\
y(0)=0 \\
y^{\prime}(1)+\varepsilon(\omega) y(1)=0 \\
\varepsilon \geq 0, x \in[0,1]
\end{array}\right.
$$

donde $\epsilon(\omega)=\mathrm{k}(\omega) / \tau$ siendo $\tau$ la tensión del hilo.

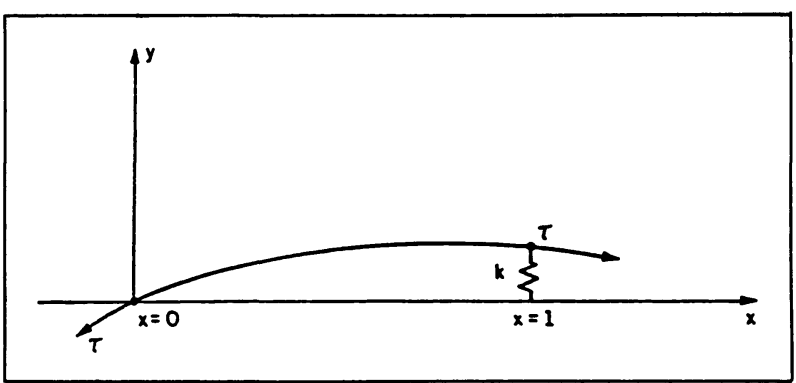

Fig. 4.11

Nuestro objetivo en este análisis es obtener aproximaciones de los primeros momentos (media y varianza) del autovalor positivo más pequeño $\lambda_{1}$. Asimismo con objeto de comparar resultados estudiamos los mismos casos planteados por Haines (v. [10]). Dicho autor considera en este problema $\mathrm{a} \epsilon$ como variable aleatoria uniforme en los intervalos siguientes:

19) $\left.\left.\left.[0,0.5], 2^{9}\right)[0,1], 3^{a}\right)[0,2], 4^{9}\right)[0,4]$,

$\left.\left.\left.5^{\circ}\right)[0,8], 6^{\circ}\right)[0,16], 7^{9}\right)[0,32]$.

La relación entre las variables $\lambda$ y $\epsilon$ puede obtenerse en forma implícita, siendo ésta:

$$
\sqrt{\lambda}=-\varepsilon \operatorname{tg} \sqrt{\lambda}
$$

La gráfica de $\lambda_{1}=g(\epsilon)$ correspondiente a la rama que da el autovalor positivo más pequeño, es (ver Fig. 4.12), donde $\mathrm{g}$ establece una correspondencia biunívoca entre el intervalo $(0,+\infty)$ de $\epsilon$ y $\left(\pi^{2} / 4, \pi^{2}\right)$ de $\lambda_{1}$.

Los momentos $\beta_{k}=E\left[\lambda_{1} k\right] ; k=0,1,2, \ldots$ pueden estimarse a partir de $\beta_{k}{ }^{*}(n)=E\left[\left(\lambda_{1}\right) n^{k}\right]=E\left[g^{k}\left(\epsilon_{n}\right)\right]$ donde $\epsilon n$ es la variable aleatoria discreta asociada a $\epsilon$ que posee los mismos momentos que dicha variable hasta el de orden $2 n-1$. 


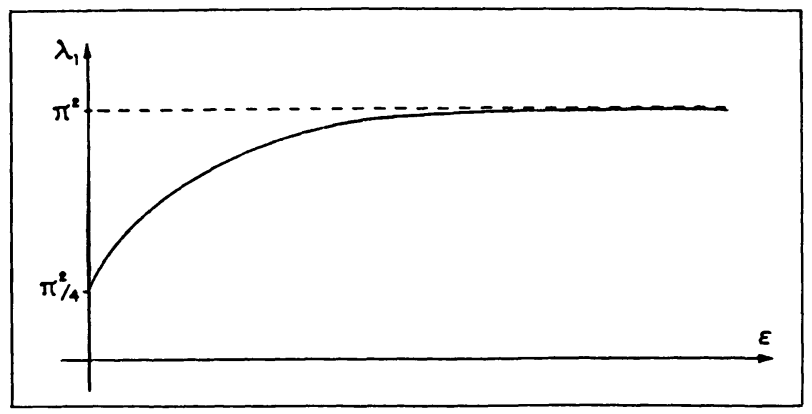

Fig. 4.12

Sea

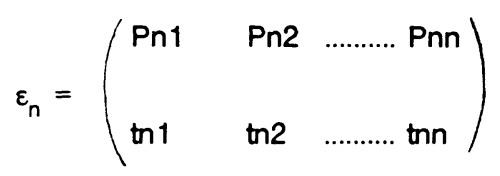

la determinación de

$$
\left(\lambda_{1}\right)_{n}=g\left(\varepsilon_{n}\right)=\left(\begin{array}{llll}
P n 1 & P n 2 & \ldots \ldots . . . . . P n n \\
g(\operatorname{tn} 1) & g(t n 2) & \ldots \ldots . . . . g(t m n)
\end{array}\right)
$$

equivale a resolver (4.4) para cada $\epsilon=$ tni ; $i=1, \ldots n$, o lo que es lo mismo en este caso, resolver la ecuación (4.5) y a asociar a cada solución la probabilidad Pni ; i $=1, \ldots, n$.

Por ser $\epsilon$ uniforme en $[0, b]$ para $b=0.5,1,2,4,8,16$ y 32 , $\epsilon_{n}$ resulta aplicando (4.1):

$$
\varepsilon_{n}=\left\{\begin{array}{l}
\text { Pni }=\quad \text { Ani } / 2 \\
\operatorname{tni}=\frac{b}{2}(Z n i+1) \\
i=1,2, \ldots n
\end{array}\right.
$$

donde Zni y Ani son los ceros y números de Christoffel relativos a los polinomios de Legendre en $[-1,1]$.

De esta forma se han obtenido los siguientes valores de la media $(M)$ y la varianza $(V)$ de $\left(\lambda_{1}\right)_{n}$, donde se ha considerado $n=2,4,6,8$ y 10 en cada uno de los siete casos (ver Tabla 4.3 en la pág. siguiente).

Por otra parte, los valores obtenidos como estimaciones de la media y la varianza de $\lambda_{1}$ mediante el método de Monte Carlo, empleando muestras de tamaño $N=100$ y 1000 y en cuatro realizaciones distintas para cada caso y tamaño muestral están recogidos en la Tabla 4.4 en la cual la media y la varianza muestral son designadas por $M^{*}$ y $V^{*}$ respectivamente.

A continuación se estiman la media y la varianza de $\lambda_{1}$ aproximando la relación $\lambda_{1}=g(\epsilon)$ por $a_{0}+a_{1}\left(\epsilon-\epsilon_{0}\right)$ $+a_{2}\left(\epsilon-\epsilon_{0}\right)^{2}$ es decir limitando el desarrollo de Tay- lor de $\mathrm{g}$ a dos y tres términos respectivamente, donde como es habitual en este método $\epsilon_{o}=E[\epsilon]$.

Sea $\lambda_{1}{ }^{*}$ la variable aleatoria función de la variable $\epsilon$ definida como

$$
\lambda_{1}{ }^{*}=g_{1}^{*}(\varepsilon)=a_{0}+a_{1}\left(\varepsilon-\varepsilon_{0}\right)
$$

y análogamente

$$
\lambda_{1}^{* *}=g 2^{*}(\varepsilon)=a_{0}+a_{1}\left(\varepsilon-\varepsilon_{0}\right)+a_{2}\left(\varepsilon-\varepsilon_{0}\right)^{2} .
$$

De esta forma en el primer caso, la esperanza es:

$$
E\left[\lambda_{1}\right]=E[g(\varepsilon)] \cong E\left[\lambda_{1}^{*}\right]=a_{\circ}
$$

y la varianza

$$
D^{2}\left[\lambda_{1}\right]=D^{2}[g(\varepsilon)] \cong D^{2}\left[\lambda_{1}^{*}\right]=a_{1}^{2} D^{2}[\varepsilon]
$$

análogamente en el segundo:

$$
\begin{aligned}
& E\left[\lambda_{1}\right] \cong E\left[\lambda_{1}^{* *}\right]=a_{0}+a_{2} D^{2}[\varepsilon] \\
& D^{2}\left[\lambda_{1}\right] \cong D^{2}\left[\lambda_{1}^{* *}\right]=a_{1}^{2} D^{2}[\varepsilon]-a_{2}^{2}\left(D^{2}[\varepsilon]\right)^{2} \\
& +2 a_{1} a_{2} E\left[\left(\varepsilon-\varepsilon_{0}\right)^{3}\right]+a_{2}^{2} E\left[\left(\varepsilon-\varepsilon_{0}\right)^{4}\right]
\end{aligned}
$$

Por otro lado como $\epsilon$ tiene distribución uniforme en [0,b] se tiene:

$$
\begin{gathered}
\varepsilon_{0}=E[\varepsilon]=b / 2, D^{2}[\varepsilon]=b^{2} / 12, \quad E\left[\left(\varepsilon-\varepsilon_{0}\right)^{3}\right]=0 y \\
E\left[\left(\varepsilon-\varepsilon_{0}\right)^{4}\right]=b^{4} / 80
\end{gathered}
$$

Los valores $a_{0}$, $a_{1}$ y a2 se han obtenido en [13] aplicando el método de perturbación (v. [7] y [10]). También pueden obtenerse en este caso a partir de la expresión:

$$
\sqrt{\lambda}=-\varepsilon \operatorname{tg} \sqrt{\lambda}
$$

derivando dicha relación implícitamente.

En la Tabla 4.5 se dan los valores $E\left[\lambda_{1}{ }^{*} b D^{2}\left[\lambda_{1}{ }^{*}\right]\right.$, $E\left[\lambda_{1}{ }^{* *}\right]$, y $D^{2}\left[\lambda_{1}{ }^{* *}\right]$ (junto con $a_{0}$ a1 y a2), los cuales pueden compararse con las correspondientes de las Tablas 4.3 y 4.4 .

A la vista de las mismas y de cada uno de los siete casos analizados, se concluye que la estimación de los momentos (media y varianza) de $\lambda_{1}$ obtenida a partir de $\left(\lambda_{1}\right)_{n}$ ( $v$. Tabla 4.3) es incluso para valores pequeños de $n(n=2$ ó 3) mejor, que la dada por el método de Taylor a partir de las variables $\lambda_{1}{ }^{*} y \lambda_{1}{ }^{* *}$ y que la dada por el método de Monte Carlo para tamaños muestrales $N=100$ y 1000 . 


\begin{tabular}{|c|c|c|c|c|c|c|c|}
\hline \multirow{2}{*}{$\mathbf{n}$} & \multicolumn{7}{|c|}{ Casos: } \\
\hline & $\left.1 .^{\circ}\right)[0,05]$ & $\left.2 .{ }^{\circ}\right)[0,1]$ & 3. $\left.{ }^{\circ}\right)[0,2]$ & 4. $\left.{ }^{\circ}\right)[0,4]$ & $\left.5 .{ }^{\circ}\right)[0,8]$ & $\left.6^{\circ}\right)[0,16]$ & $\left.7 .^{\circ}\right)[0,32]$ \\
\hline 2 & $\begin{array}{l}M=2.9353956500 \\
V=0.0681860169\end{array}$ & $\begin{array}{c}3.34600192 \\
0.224210559\end{array}$ & $\begin{array}{l}4.029315910 \\
0.615835733\end{array}$ & $\begin{array}{l}5.01625637 \\
1.24310644\end{array}$ & $\begin{array}{l}6.201416220 \\
1.605400310\end{array}$ & $\begin{array}{l}7.38278558 \\
1.23678990\end{array}$ & $\begin{array}{l}8.361794140 \\
0.597717853\end{array}$ \\
\hline 4 & $\begin{array}{l}M=2.9353940300 \\
V=0.0683003365\end{array}$ & $\begin{array}{l}3.345975570 \\
0.225695463\end{array}$ & $\begin{array}{l}4.028924530 \\
0.631668951\end{array}$ & $\begin{array}{l}5.01184174 \\
1.35903958\end{array}$ & $\begin{array}{l}6.172047480 \\
2.087228690\end{array}$ & $\begin{array}{l}7.28381786 \\
2.26365580\end{array}$ & $\begin{array}{l}8.189092260 \\
1.732911650\end{array}$ \\
\hline 6 & $\begin{array}{l}M=2.9353929300 \\
V=0.0683013330\end{array}$ & $\begin{array}{l}3.345973400 \\
0.225698974\end{array}$ & $\begin{array}{l}4.028920260 \\
0.631681034\end{array}$ & $\begin{array}{l}5.01183467 \\
1.35910194\end{array}$ & $\begin{array}{l}6.172051180 \\
2.089949910\end{array}$ & $\begin{array}{l}7.28289459 \\
2.31035777\end{array}$ & $\begin{array}{l}8.178375110 \\
1.950842820\end{array}$ \\
\hline 8 & $\begin{array}{l}M=2.9353949700 \\
V=0.0683005619\end{array}$ & $\begin{array}{l}3.345977260 \\
0.225696049\end{array}$ & $\begin{array}{l}4.028927220 \\
0.631670581\end{array}$ & $\begin{array}{l}5.01184650 \\
1.35906787\end{array}$ & $\begin{array}{l}6.172070460 \\
2.089848880\end{array}$ & $\begin{array}{l}7.28294030 \\
2.31054419\end{array}$ & $\begin{array}{l}8.178219390 \\
1.967346980\end{array}$ \\
\hline 10 & $\begin{array}{l}M=2.9353940200 \\
V=0.0683003878\end{array}$ & $\begin{array}{l}3.345975570 \\
0.225695451\end{array}$ & $\begin{array}{l}4.028924540 \\
0.631668823\end{array}$ & $\begin{array}{l}5.01184299 \\
1.35906398\end{array}$ & $\begin{array}{l}6.172066980 \\
2.089843520\end{array}$ & $\begin{array}{l}7.28293776 \\
2.31053073\end{array}$ & $\begin{array}{l}8.178233660 \\
1.967857080\end{array}$ \\
\hline
\end{tabular}

Tabla 4.3

\begin{tabular}{|c|c|c|c|c|c|c|c|c|}
\hline & \multicolumn{8}{|c|}{ Casos: } \\
\hline & $\begin{array}{c}\text { Realización } \\
\text { Muestral }\end{array}$ & $\left.1 . .^{\circ}\right)[0,5]$ & $\left.2 .^{\circ}\right)[0,1]$ & 3. $\left.{ }^{\circ}\right)[0,2]$ & 4..$\left.^{\circ}\right)[0,4]$ & $\left.5 .^{\circ}\right)[0,8]$ & $\left.6 .^{\circ}\right)[0,16]$ & $7^{\circ} .^{\circ}[0,32]$ \\
\hline \multirow{4}{*}{$\begin{array}{l}8 \\
\stackrel{8}{1} \\
\text { II } \\
\mathbf{z}\end{array}$} & 1 & $\begin{array}{l}M^{*}=2.9559683500 \\
V^{*}=0.0610716578\end{array}$ & $\begin{array}{l}3.384044580 \\
0.191744461\end{array}$ & $\begin{array}{l}4.070718380 \\
0.548463698\end{array}$ & $\begin{array}{l}4.9717115900 \\
1.0597627600\end{array}$ & $\begin{array}{l}6.37692242 \\
1.62241019\end{array}$ & $\begin{array}{l}7.41362670 \\
2.23950043\end{array}$ & $\begin{array}{l}8.41818283 \\
1.31328675\end{array}$ \\
\hline & 2 & $\begin{array}{l}M^{*}=2.9462592700 \\
V^{*}=0.0702575087\end{array}$ & $\begin{array}{l}3.327506680 \\
0.234940378\end{array}$ & $\begin{array}{l}4.061782460 \\
0.531923637\end{array}$ & $\begin{array}{l}4.9648815700 \\
1.4032398700\end{array}$ & $\begin{array}{l}6.02261596 \\
1.91034994\end{array}$ & $\begin{array}{l}7.36654365 \\
2.56863004\end{array}$ & $\begin{array}{l}8.36529290 \\
1.33106508\end{array}$ \\
\hline & 3 & $\begin{array}{l}M^{*}=2.9185826400 \\
V^{*}=0.0715346071\end{array}$ & $\begin{array}{l}3.362688290 \\
0.189839929\end{array}$ & $\begin{array}{l}3.887270040 \\
0.665355362\end{array}$ & $\begin{array}{c}5.0675284700 \\
1.180684720\end{array}$ & $\begin{array}{l}6.09106280 \\
2.31828383\end{array}$ & $\begin{array}{l}7.38836839 \\
2.49236587\end{array}$ & $\begin{array}{c}8.10117096 \\
2.0685177\end{array}$ \\
\hline & 4 & $\begin{array}{l}M^{*}=2.8811454400 \\
V^{*}=0.0608284240\end{array}$ & $\begin{array}{l}3.341727090 \\
0.236864892\end{array}$ & $\begin{array}{l}4.049918760 \\
0.696047703\end{array}$ & $\begin{array}{l}5.1373204211 \\
1.0194271100\end{array}$ & $\begin{array}{l}6.10312547 \\
2.39848784\end{array}$ & $\begin{array}{l}7.48961713 \\
1.63529215\end{array}$ & $\begin{array}{l}8.42051361 \\
1.09978309\end{array}$ \\
\hline \multirow{4}{*}{$\begin{array}{l}8 \\
\varnothing \\
11 \\
z\end{array}$} & 1 & $\begin{array}{l}M^{*}=2.9362122600 \\
V^{*}=0.0701108810\end{array}$ & $\begin{array}{l}3.362884640 \\
0.232071276\end{array}$ & $\begin{array}{l}4.045947370 \\
0.645765322\end{array}$ & $\begin{array}{l}5.11207669 \\
1.30674477\end{array}$ & $\begin{array}{l}6.18932648 \\
2.00102429\end{array}$ & $\begin{array}{l}7.26536040 \\
2.19518381\end{array}$ & $\begin{array}{l}8.12537113 \\
2.03372044\end{array}$ \\
\hline & 2 & $\begin{array}{l}M^{*}=2.9379029700 \\
V^{*}=0.0667478514\end{array}$ & $\begin{array}{l}3.326283840 \\
0.226541097\end{array}$ & $\begin{array}{l}4.053769500 \\
0.636027567\end{array}$ & $\begin{array}{l}5.08286859 \\
1.36184219\end{array}$ & $\begin{array}{l}6.14966540 \\
2.09604699\end{array}$ & $\begin{array}{l}7.22886524 \\
2.37743394\end{array}$ & $\begin{array}{l}8.19782922 \\
2.00586470\end{array}$ \\
\hline & 3 & $\begin{array}{l}M^{*}=2.9375459500 \\
V^{*}=0.0683700530\end{array}$ & $\begin{array}{l}3.337940120 \\
0.238839040\end{array}$ & $\begin{array}{l}4.038976490 \\
0.642732068\end{array}$ & $\begin{array}{l}5.04322843 \\
1.33364961\end{array}$ & $\begin{array}{l}6.16367922 \\
2.08247058\end{array}$ & $\begin{array}{l}7.23765458 \\
2.32533484\end{array}$ & $\begin{array}{l}8.13232718 \\
2.09853337\end{array}$ \\
\hline & 4 & $\begin{array}{l}M^{*}=2.9468641200 \\
V^{*}=0.0682063887\end{array}$ & $\begin{array}{l}3.367729310 \\
0.217690103\end{array}$ & $\begin{array}{l}4.040332920 \\
0.646998732\end{array}$ & $\begin{array}{l}4.98920235 \\
1.36447839\end{array}$ & $\begin{array}{l}6.21235513 \\
1.96537427\end{array}$ & $\begin{array}{l}7.27107112 \\
2.40916126\end{array}$ & $\begin{array}{l}8.21378292 \\
1.69494336\end{array}$ \\
\hline
\end{tabular}




\begin{tabular}{|c|c|c|c|c|c|c|c|}
\hline & \multicolumn{7}{|c|}{ Casos: } \\
\hline & 1. $\left.{ }^{\circ}\right)[0,05]$ & $\left.2 .^{\circ}\right)[0,1]$ & $\left.3^{\circ}{ }^{\circ}\right)[0,2]$ & 4. $\left.{ }^{\circ}\right)[0,4]$ & $\left.5^{\circ}\right)[0,8]$ & 6. $\left.{ }^{\circ}\right)[0,16]$ & 7..$\left.^{\circ}\right)[0,32]$ \\
\hline$a_{o}$ & 2.9429647900 & 3.373089290 & 4.115858380 & 5.239199310 & 6.6071184100 & 7.8648003800 & 8.7541258300 \\
\hline$a_{1}$ & 1.8080151500 & 1.636195130 & 1.345962620 & 0.932308283 & 0.4966429140 & 0.1969528580 & 0.0623615111 \\
\hline$a_{2}$ & -0.3632217490 & -0.324651535 & -0.258146759 & -0.163095408 & -0.0699653853 & -0.0187384385 & $-3.4498082600 \times 10^{-3}$ \\
\hline$E\left[\lambda_{1}{ }^{*}\right]$ & 2.942964800 & 3.373089290 & 4.115858380 & 5.239199300 & 6.6071184100 & 7.8648003800 & 8.7541258300 \\
\hline$D^{2}\left[\lambda_{1}{ }^{*}\right]$ & 0.0681024748 & 0.223094542 & 0.603871792 & 1.158931650 & 1.3154889800 & 0.8275291360 & 0.3318577550 \\
\hline$E\left[\lambda_{1}{ }^{\star *}\right]$ & 2.9353976800 & 3.346035000 & 4.029809460 & 5.021738760 & 6.2339696900 & 7.4650470300 & 8.4597421900 \\
\hline$D^{2}\left[\lambda_{1}{ }^{* *}\right]$ & 0.0681482838 & 0.223680090 & 0.609795326 & 1.196762920 & 1.4268809600 & 0.9553713320 & 0.4011871360 \\
\hline
\end{tabular}

\section{Comentarios y conclusiones}

El procedimiento expuesto tiene en común con el método de Monte Carlo el hecho de dar lugar, en ambos casos, a la resolución de una familia de problemas deterministas. Precisamente fue esta idea la que se tomó como punto de partida en la construcción del método.

Sin embargo, la diferencia fundamental entre ambos métodos es que con el método propuesto la convergencia a la solución (momentos, función de distribución de la variable en cuestión) es de tipo determinista, es decir aumentando el número de puntos de discretización, el error cometido en la estimación decrece. Por otro lado la aplicación de la simulación de Monte Carlo da lugar a unos valores para los cuales la probabilidad de que difieran de los teóricos en una cantidad mayor que $\epsilon>0$ (con $\epsilon$ arbitrario), decrece, tendiendo a cero, cuando el tamaño de la muestra tiende a infinito. Es decir, aquí lo que decrece es la probabilidad de que el error cometido en la estimación sea mayor que un valor $\epsilon$ dado.

Esta diferencia de esquemas se ha podido observar en los ejemplos expuestos para los cuales los valores obtenidos a partir de una muestra de tamaño dado dependian en cierta medida de la realización muestral. Sin embargo con el método propuesto y empleando un número pequeño de puntos de discretización, la estimación conseguida resulta muy aceptable, pudiendo asegurar un cierto número de cifras del resultado. Téngase en cuenta que para que los resultados obtenidos por el método de Monte Carlo tengan un cierto grado de fiabilidad, el tamaño de la muestra debe ser en general $\mathrm{N} \geq 1000$.

Por otra parte se ha de indicar que una de las grandes ventajas del método de Monte Carlo: "generar muestras de cualquier tamaño N", puede también conseguirse en parte con el método propuesto en la siguiente forma:

Sea $Y=g\left(X_{1}, X_{2}, \ldots X_{q}\right)$; la determinación de momentos, función de distribución, etc. de $Y$ requiere como ya se ha dicho la resolución de $N=n_{1} \times n_{2} \times \ldots . . \times n_{q}$ problemas (se supone que $g$ no es conocida explícitamente). Si se desea que $\mathrm{N}$ no sea grande (para competir económicamente con el método de Monte Carlo) será necesario discretizar cada variable $X_{i} ; i=1, \ldots q$ en un número pequeño de puntos $n_{i} ; i=1, \ldots q$, incluso será obligado hacer en muchas de las variables $\mathrm{n}_{\mathrm{i}}=1$ cuando $\mathrm{q}$ sea grande. Esto corresponde a considerar para dichas variables el valor medio es decir:

$$
X_{1}^{(i)}=\left(\begin{array}{l}
p_{11}^{(i)} \\
t_{11}^{(i)}
\end{array}\right)=\left(\begin{array}{l}
1 \\
E\left[X_{i}\right]
\end{array}\right)
$$

lo que equivale en definitiva a tomar como deterministas dichas variables. Un criterio para determinar estas variables puede ser elegir aquellas que contribuyan lo menos posible a la varianza de $Y$.

También destacamos la posibilidad de desarrollar una técnica de Monte Carlo a partir del método propuesto. Para ello basta considerar las distribuciones discretas

$$
F_{X n_{1}}^{(i)}
$$


asociadas a cada variable $X_{i} ; i=1, \ldots q$. De esta manera, una muestra de tamaño $\mathrm{N}$ está formada por $\mathrm{N}$ q -uplas

$$
\left(\operatorname{tn}_{1}^{(1)}, i_{1}(j), \operatorname{tn}_{2}^{(2)}, i_{2}(j), \ldots, \operatorname{tn}_{q}^{(q)}, i_{q}(j)\right) ; j=1, \ldots N
$$

donde cada

$$
\operatorname{tn}_{\alpha}^{(\alpha)}, i_{\alpha}(j)
$$

representa para cada $\mathrm{j}=1, \ldots \mathrm{N}$ un valor al azar de entre

$$
\left\{\operatorname{tn}_{\alpha}^{(\alpha)}, 1, \operatorname{tn}_{\alpha}^{(\alpha)} 2 \ldots, \operatorname{tn}_{\alpha}^{(\alpha)} n_{\alpha}\right\}
$$

obtenido mediante la distribución

$$
F_{X_{n_{\alpha}}}^{(\alpha)} ; \alpha=1, \ldots, q
$$

De esta forma, las características estocásticas de la variable $Y=g\left(X_{1}, X_{2}, \ldots, X_{q}\right)$ pueden ser estimadas a partir de los valores de la muestra:

$$
\left\{y_{j}=g\left(t_{1}^{(1)}, i_{1}(j), \ldots, t_{q}^{(q)}, i_{q}(j)\right) ; j=1, \ldots N\right\}
$$

Un esquema probabilístico análogo a éste ha sido propuesto por Hammersley (v. Davis y Rabinowitz [8]) para reducir el problema de la dimensionalidad en la evaluación de integrales múltiples utilizando una fórmula de cuadratura producto tipo Gauss.

En cuanto a las conclusiones, podemos destacar que se ha concretado un nuevo método que permite la resolución aproximada de problemas estocásticos de muy diferente tipo (en [13] se desarrolla también una aplicación del mismo a problemas de Pandeo Estocástio, la cual se expondrá en un próximo artículo de Informes de la Construcción).

Consideramos que la utilización del mismo resulta muy adecuada en aquellos casos en los que la parte estocástica del problema está dada mediante variables aleatorias independientes o reducibles a independientes mediante una transformación.

Asimismo, en aquellos casos en los que el número de variables aleatorias que intervienen como coeficientes y/o parámetros del problema estocástico sea reducido, la aplicación del método propuesto es preferible a la de Monte Carlo ya que discretizando cada variable aleatoria en un número pequeño de puntos se obtienen en general aproximaciones muy aceptables de los primeros momentos de la variable aleatoria incógnita.

Obsérvese que el cálculo de los momentos de la variable aleatoria incógnita podria haberse planteado direc(c) Consejo Superior de Investigaciones Científicas Licencia Creative Commons 3.0 España (by-nc) tamente, aproximando las integrales mediante fórmulas de cuadratura producto tipo Gauss, llegándose evidentemente a los mismos resultados. Sin embargo el enfoque dado aqui interpretando los ceros y números de Christoffel en términos de una variable aleatoria discreta, ha permitido la utilización del lenguaje estadístico desde el principio lo cual nos ha llevado a su vez y de forma natural al concepto de variable aleatoria discreta asociada a la variable aleatoria incógnita, siendo asimismo inmediata la construcción de su función de distribución, hecho que no resulta evidente desde el planteamiento anterior.

Por otro lado hay que indicar que el método aportado puede ser también utilizado como "patrón" y como alternativo al de Monte Carlo, ya que en caso de convergencia, la discretización de cada variable aleatoria en un número creciente de puntos permite aproximar tanto como se desee la función de distribución y los momentos de la variable aleatoria incógnita, sin los inconvenientes de depender de la realización muestral.

También se ha de indicar que el citado método puede aplicarse en combinación con otros, discretizando sólo algunas de las variables aleatorias inmersas en el problema, e incluso todas si hubiese procesos estocásticos interviniendo también como coeficientes o parámetros. De esta forma al problema estocástico resultante de considerar cada combinación de valores de las variables discretizadas, se le puede aplicar otro método de resolución, asociando a su resultado la correspondiente probabilidad.

De todo lo anterior es posible concluir finalmente que el método de discretización propuesto representa un cierto avance en el campo de la Estadística Aplicada al poder resolver mediante el mismo y con una metodología diferente a la de los procedimientos actuales, muchos de los problemas estocásticos que se presentan en la Técnica, y particularmente en la Ingeniería Civil.

\section{Referencias}

1 ANG. A.H.S and TANG, W.H. (1975) "Probability Concepts in Engineering Planning and Desing". Vol. 1, John Wiley \& Sons, New York.

2 ANG. A.H.-S and TANG, W.H. (1984) "Probability Concepts in Engineering Planning and Desing". Vol. 2, John Wiley \& Sons, New York.

3 BENJAMIN, J.R. and CORNELL, C.A. (1970) "Probability, Statistics and Decision for Civil Engineers". McGraw Hill, New York.

4 BHARUCHA-REID, A.T. (1970) "Probabilistic Methods in Applied Mathematics". Vol. 2, Academic Press New York and London.

5 BHARUCHA-REID, A.T. (1972) "Random Integral Equations". Academic Press, New York and London.

http://informesdelaconstruccion.revistas.csic.es 
6 BHARUCHA-REID, A.T. (1979) "Approximate Solution of Random Equations". North Holland, New York-Oxford.

7 BOYCE, W.E. (1968) "Random eigenvalue problems". Probabilistic Methods in Applied Mathematics, Vol. 1. Bharucha-Reid, A.T. (ed) Academic Press. New York, pp 1.73.

8 DAVIS, P.J. and RABINOWITZ, P. (1984) "Method of Numerical Integration". 2 nd edition, Academic Press, New York.

9 GOLUB, G.H. and WELSCH, J.H. (1969) "Calculation of Gauss quadrature rules" Math. Comp. 23, pp. 221-230.

10 HAINES, C.W. (1965) "An analysis of stochastic eigenvalue problems". Ph. D. Thesis, Rensselaer Polytechnic Institute, Troy, New York.

11 LOEVE, M. (1976) "Teoría de la Probabilidad". 3. a edición, Tecnos, S. A. Madrid.
12 RAMDAS BHAT, B. (1981) "Modern Probability Theory". Wiley Eastern Limited, New Delhi.

13 ROMERO, J.L. (1989) "Tratamiento numérico de algunos problemas estocásticos. Contribución al estudio del pandeo estocástico". Tesis Doctoral, Universidad Politécnica de Madrid.

14 ROSENBLUETH, E. (1975) "Point estimates for probability moments" Proc. Nat. Acad, Sci. USA, Vol. 72, N. ${ }^{\circ} 10$, pp 3812-3814.

15 SCHEIDT, J. and PURKERT, W (1983) "Random Eigenvalue Problems". North Holland, New York - Amsterdam Oxford.

16 STOER, J. and BULIRSCH, R. (1980) "Introduction to Numerical Analisis". Springer-Verlag, New York.

17 STROUD, A.H. and SECRET, D. (1966) "Gaussuan Quadrature Formulas". Prentice-Hall, Englewood Cliffs, New Jersey.

\section{publicaciones del ICCET/CSIC}

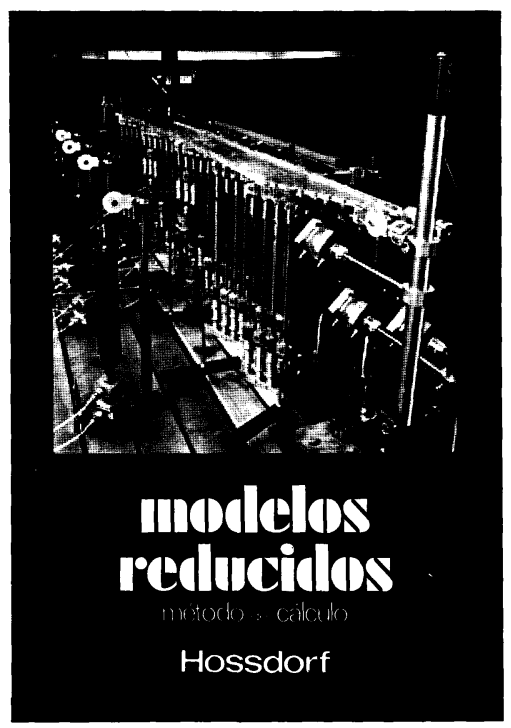

Modelos reducidos. Método de cálculo

H. Hossdorf, Ingeniero Civil

La técnica de los ensayos en modelos reducidos de estructuras sufre hoy dia una decisiva metamortosis. Hasta hace poco era un medio más bien de artesania, que no siempre era tomado en serio por los academicos teorizantes para comprender el comportamiento resistente de las estructuras complejas y al que se acudió las más de las veces, como a un último remedio debido a sus indiscutibles insuficiencias. Sin embargo, en poco tiempo y gracias a su conexión con los ordenadores digitales, se ha transformado en un instrumento cientificamente valioso, que no puede quedar a un lado en la práctica diaria del Ingeniero Proyectista.

Un volumen encuadernado en cartoné plastificado con lomo de tela, de $17 \times 24 \mathrm{~cm}$, compuesto de 250 páginas, 158 figuras y fotografias.

Precios: 1.800 ptas.; \$ USA 26.00.

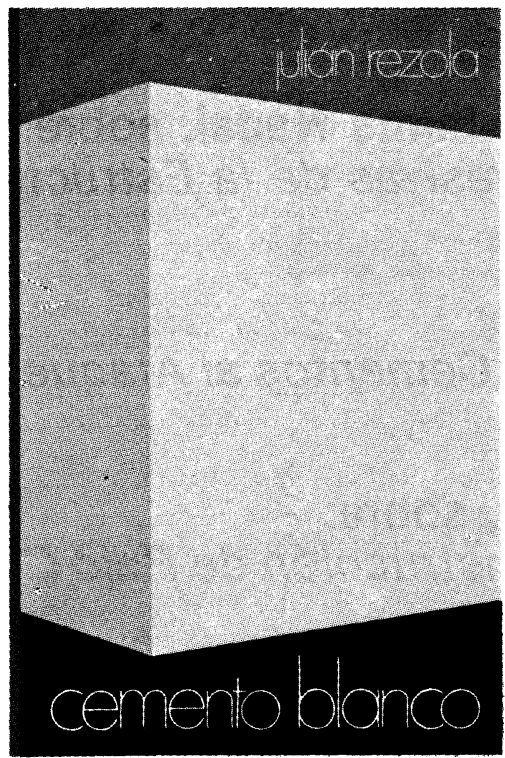

Cemento blanco

Julián Rezola

Ingeniero Químico Dipl. I. O. S.

Sabido es que existe una extensa y documentada bibliografia sobre el cemento gris: en cambio, no puede decirse lo mismo acerca del cemento portlan blanco, ya que los escritos existentes se refieren tan sólo a algunas peculiaridades que le distinguen de aquél.

El autor nos ofrece sus profundos conocimientos y su larga experiencia tanto en laboratorio como en fabricación.

La parte descriptiva del libro se complementa con gráficos, diagramas y fotografias de gran utilidad grácicos, diagramas y fologras de gran utilidad, cste aglomeranter.

Un volumen encuadernado en cartoné policerado, de $17,4 \times 24,3 \mathrm{~cm}$, compuesto de 395 páginas, numerosas figuras, tablas y ábacos.

Precios: España, 1.700 ptas.; extranjero, \$ 24.

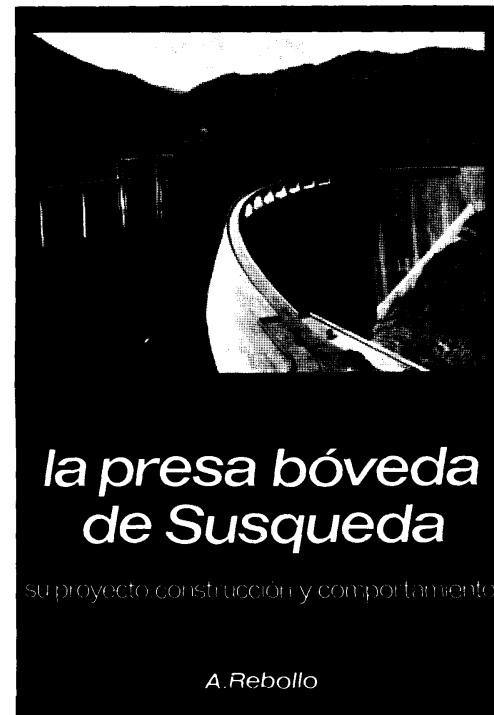

La presa bóveda de Susqueda

A. Rebollo,

Dr. Ingeniero de Caminos

El esfuerzo del constructor de presas se sitúa por su pretensión de perennidad, a contracorriente de las tendencias de la civilización actual, caracterizada por to fungible. Pueden evocarse las 10.000 grandes. presas en funcionamiento o en construcción gue prén que estón gerontológicos para manter y peffeccionar su peren id gn lizar mu inalenable preccionsión de perencidad. En la medida en que todas nuevas riesgo grandes o pequeñas, son portadoras de aumentan con el envejecimiento, la gerolicos, que aumen las presas cos todo un emplazo. La acción adelantada a Artur Rebollo un este tereno marca un camino a devoción paterna que al ha puesto en Susqueda.

Un volumen encuadernado en cartoné plastificado con lomo encuadernado en cartone plastificado 408 páginas, 330 figuras y fotografias y 39 tablas. Precios: 1.700 ptas.; extranjero, \$ USA 24.00 . 